\title{
Morphology Transcription in Hierarchical MOF-on-MOF Architectures
}

Kun-Yu Wang ${ }^{\dagger}$, Liang Feng ${ }^{\dagger *}$, Tian-Hao Yan ${ }^{\dagger}$, Jun-Sheng Qin', Chen-Xuan $\mathrm{Li}^{\dagger}$, and Hong-Cai Zhou ${ }^{\dagger * *}$

${ }^{\dagger}$ Department of Chemistry, Texas A\&M University, College Station, Texas 77843-3255, United

States

*Department of Materials Science and Engineering, Texas A\&M University, College Station,

Texas 77843-3003, United States

' College of Chemistry, Jilin University, Changchun, 130012, China

Corresponding authors: fengliang@tamu.edu; zhou@chem.tamu.edu.

\section{Table of Contents}

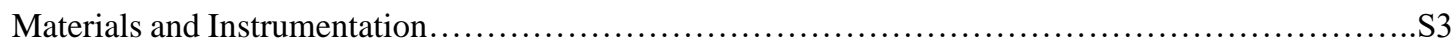

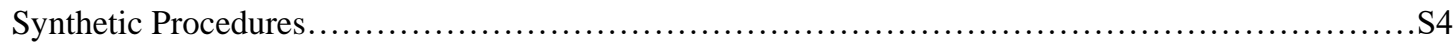

Figure S1 Illustration of the decomposition of $\mathrm{Zn}_{2}(\mathrm{BDC})_{2}(\mathrm{TED})$ under Na-EDTA treatment........S5

Figure S2 Stepwise polymerization and template removal processes in $\mathrm{Zn}_{2}(\mathrm{BDC})_{2}(\mathrm{TED}) \ldots \ldots \ldots \ldots . \mathrm{S} 6$

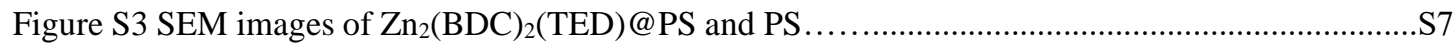

Figure S4 Stepwise polymerization and template removal processes in PCN-222@ $\mathrm{Zn}_{2}(\mathrm{BDC})_{2}(\mathrm{TED})$

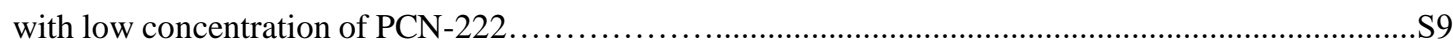

Figure S5 Stepwise polymerization and template removal processes in hierarchical PCN-

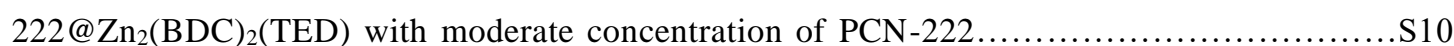

Figure S6 Stepwise polymerization and template removal processes in hierarchical PCN$222 @ \mathrm{Zn}_{2}(\mathrm{BDC})_{2}(\mathrm{TED})$ with high concentration of PCN-222.

Figure S7 Stepwise polymerization and template removal processes in hierarchical PCN-222 nanorods@ $\mathrm{Zn}_{2}(\mathrm{BDC})_{2}(\mathrm{TED})$.

Figure S8 Stepwise polymerization and template removal processes in hierarchical PCN-222 microneedles@ $\mathrm{Zn}_{2}(\mathrm{BDC})_{2}(\mathrm{TED})$

Figure S9 Tunable sizes of PCN-222 in hierarchical MOF-on-MOF and MOF-on-polymer architectures

Figure S10 Decomposition of PCN-222@ $\mathrm{Zn}_{2}(\mathrm{BDC})_{2}(\mathrm{TED})$ under Na-EDTA treatment.... S15

Figure S11. ${ }^{1} \mathrm{H}$ NMR spectroscopy of digested $\mathrm{Zn}_{2}(\mathrm{BDC})_{2}(\mathrm{TED})$ S16

Figure $\mathrm{S} 12{ }^{1} \mathrm{H}$ NMR spectroscopy of templated PS S16

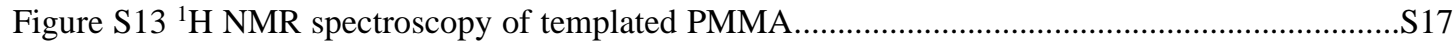

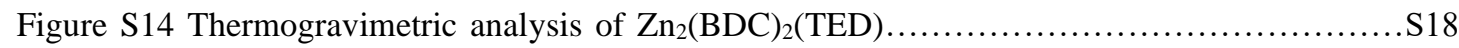

Figure S15 Thermogravimetric analysis of templated PS ...................................... 18

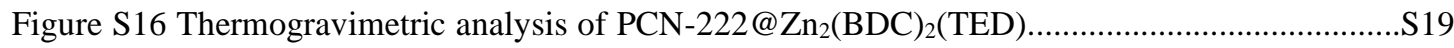


Figure S17 Thermogravimetric analysis of templated PCN-222@PS

Figure S18 Stepwise polymerization and template removal processes in $\mathrm{Zn}_{2}(\mathrm{BDC})_{2}(\mathrm{TED})$ to $\mathrm{Zn}_{2}(\mathrm{BDC})_{2}$ (TED)@PMMA, to templated PMMA...

Figure S19 Stepwise polymerization and template removal processes from hierarchical PCN$222 @ \mathrm{Zn}_{2}(\mathrm{BDC})_{2}(\mathrm{TED})$ to PCN-222@ $\mathrm{Zn}_{2}(\mathrm{BDC})_{2}$ (TED)@PMMA, to PCN-222@PMMA. S20

Figure S20 Stepwise polymerization and template removal processes in hierarchical PCN$160 @ \mathrm{Zn}_{2}(\mathrm{BDC})_{2}(\mathrm{TED})$

Figure S21 Stepwise polymerization and template removal processes in hierarchical PCN$224 @ \mathrm{Zn}_{2}(\mathrm{BDC})_{2}(\mathrm{TED})$

Figure S22 SEM images of PCN-224@ $\mathrm{Zn}_{2}(\mathrm{BDC})_{2}(\mathrm{TED}) @ \mathrm{PS}$ S25

Figure S23 SEM images of PCN-224@PS after template removal.... S25

Figure S24 SEM images, the corresponding EDS mapping and EDS spectrum of PCN$224 @ \mathrm{Zn}_{2}(\mathrm{BDC})_{2}(\mathrm{TED}) @ \mathrm{PS}$. S26

Figure S25 SEM images, the corresponding EDS mapping and EDS spectrum of PCN-224@PS........S26

Figure S26 Stepwise polymerization and template removal processes in hierarchical MOF$801 @ \mathrm{Zn}_{2}(\mathrm{BDC})_{2}(\mathrm{TED})$ .S28

Figure S27 Stepwise polymerization and template removal processes in hierarchical PCN$900 @ \mathrm{Zn}_{2}(\mathrm{BDC})_{2}(\mathrm{TED})$ S30

Figure S28 Stepwise polymerization and template removal processes in hierarchical COF$303 @ \mathrm{Zn}_{2}(\mathrm{BDC})_{2}(\mathrm{TED})$

Figure S29. Gel permeation chromatogram of the synthesized PS. S32

Figure S30. Nitrogen sorption tests and pore size distribution of MOFs, MOF@polymer composites, and polymers. S34

Table S1. The calculated number average molar mass Mn and dispersity ( $Đ$ ) values of PS produced from different templating MOFs.

Table S2. Elemental contents in the MOF@polymer composite materials determined by ICP-MS...S35 


\section{Materials and Instrumentation}

All reagents and solvents were commercially available and used as received. Vinyl monomers (St and MMA) were purified by vacuum distillation prior to use.

Powder X-ray diffraction (PXRD). PXRD was carried out with a Bruker D8Focus Bragg-Brentano X-ray Powder Diffractometer equipped with a $\mathrm{Cu}$ sealed tube $(\lambda$ $=1.54178 \AA$ ) at $40 \mathrm{kV}$ and $40 \mathrm{~mA}$.

${ }^{1}$ H NMR spectroscopy. Nuclear magnetic resonance (NMR) data were collected on an Avance Neo 400 spectrometer. For ${ }^{1} \mathrm{H}$ NMR spectroscopy, the activated samples (around $10 \mathrm{mg}$ ) were dissolved in $600 \mu \mathrm{L} \mathrm{DMSO}-d_{6}$ solution containing $20 \mu \mathrm{L} \mathrm{HF}$.

Thermogravimetric analysis (TGA). About $10 \mathrm{mg}$ of the sample was heated on a TGA Q500 thermogravimetric analyzer from room temperature to $600{ }^{\circ} \mathrm{C}$ at a rate of $5^{\circ} \mathrm{C} \cdot \mathrm{min}^{-1}$ under air flow of $20 \mathrm{~mL} \cdot \mathrm{min}^{-1}$.

Scanning electron microscopy (SEM). Images and analyses of SEM/EDX were taken by FEI Quanta 600 FE-SEM. The Quanta 600 FEG is a field emission scanning electron microscope capable of generating and collecting high-resolution and lowvacuum images. Source: Field emission gun assembly with Schottky emitter source. Voltage: $200 \mathrm{~V}$ to $30 \mathrm{kV}$. Beam Current: $>100 \mathrm{nA}$.

ICP-MS analysis. Calibration standards were prepared from certified reference standards from RICCA Chemical Company. Samples were further analyzed with a Perkin Elmer NexION ${ }^{\circledR}$ 300D ICP-MS.

Size exclusive chromatography (SEC). SEC was performed on a $\mathrm{TOSOH}$ EcoSEC (HLC-8320GPC) chromatography at $40{ }^{\circ} \mathrm{C}$ with UV detector at $254 \mathrm{~nm}$ and THF as the eluent. The molecular weights were calculated using a calibration curve based on the UV absorption signal of polystyrene standards. 


\section{Synthetic Procedures}

Synthesis of $\mathbf{Z n}_{2}$ (BDC) 2 (TED). $\mathrm{Zn}_{2}(\mathrm{BDC})_{2}$ (TED) (BDC = 1,4-benzenedicarboxylate, $\mathrm{TED}=$ triethylenediamine) was synthesized on the basis of previous reports with slight modifications. ${ }^{[1]}$ A solution of TED $(0.028 \mathrm{~g})$ in $N, N$-dimethylformamide (DMF, $3 \mathrm{~mL}$ ) was transferred into a mixture of $\mathrm{Zn}\left(\mathrm{NO}_{3}\right)_{2} \cdot 9 \mathrm{H}_{2} \mathrm{O}(0.150 \mathrm{~g})$ and $\mathrm{BDC}(0.084 \mathrm{~g})$ in DMF $(3 \mathrm{~mL})$. The resultant mixture was stirred for $3 \mathrm{~h}$ at room temperature, and was filtered to remove white precipitates. The filtrate was kept standing at $100{ }^{\circ} \mathrm{C}$ for 2 days. The solution was decanted, and cubic crystals were three times washed with DMF and tetrahydrofuran (THF), respectively.

\section{Polymerization of vinyl monomers in $\mathrm{Zn}_{2}(\mathrm{BDC})_{2}$ (TED) followed by template} removal. The dried host compound $\mathrm{Zn}_{2}(\mathrm{BDC})_{2}$ (TED) (100 mg) was prepared by evacuation $(<0.1 \mathrm{kPa})$ at $130{ }^{\circ} \mathrm{C}$ for 1 day in a Pyrex reaction tube. Subsequently, $\mathrm{Zn}_{2}$ (BDC) $)_{2}$ (TED) was immersed in a purified monomer $(0.5 \mathrm{~mL})$ with AIBN $(3 \mathrm{mg})$ at room temperature for $0.5 \mathrm{~h}$ to incorporate the monomer and the radical initiator into the nanochannels. After excess monomer external to the host crystals was removed completely by evacuation $(0.2 \mathrm{kPa})$ at room temperature for $0.5 \mathrm{~h}$, the reaction tube was filled with nitrogen, and heated to $70{ }^{\circ} \mathrm{C}$ to perform the polymerization of styrene for $48 \mathrm{~h}$. To isolate the polymer inside the channels, the composite was stirred for 4 days in a $0.05 \mathrm{M}$ aqueous solution $(40 \mathrm{~mL})$ of sodium ethylenediaminetetraacetate (NaEDTA) for the complete dissolution of the frameworks of $\mathrm{Zn}_{2}$ (BDC) $)_{2}$ (TED). The collected polymer was washed with water and dried under a reduced pressure at room temperature. 

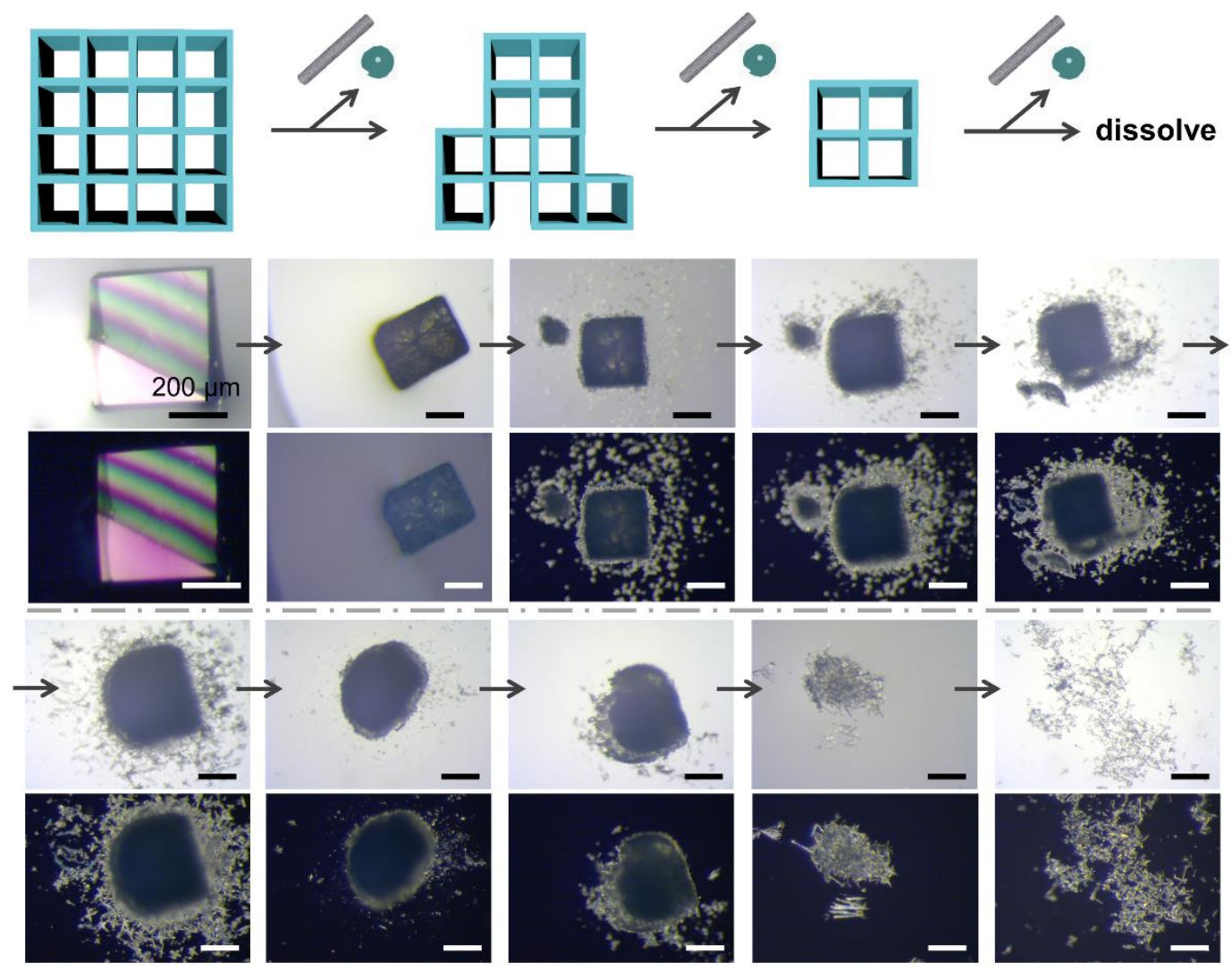

Figure S1. Illustration of the decomposition of $\mathrm{Zn}_{2}(\mathrm{BDC})_{2}(\mathrm{TED})$ under Na-EDTA treatment. Optical images and polarized optical images of $\mathrm{Zn}_{2}(\mathrm{BDC})_{2}(\mathrm{TED})$ crystals treated with Na-EDTA solutions shown fast decomposition of the framework when in the absence of polymerization. (Cyan frameworks $=\mathrm{Zn}_{2}(\mathrm{BDC})_{2}$ (TED)) 

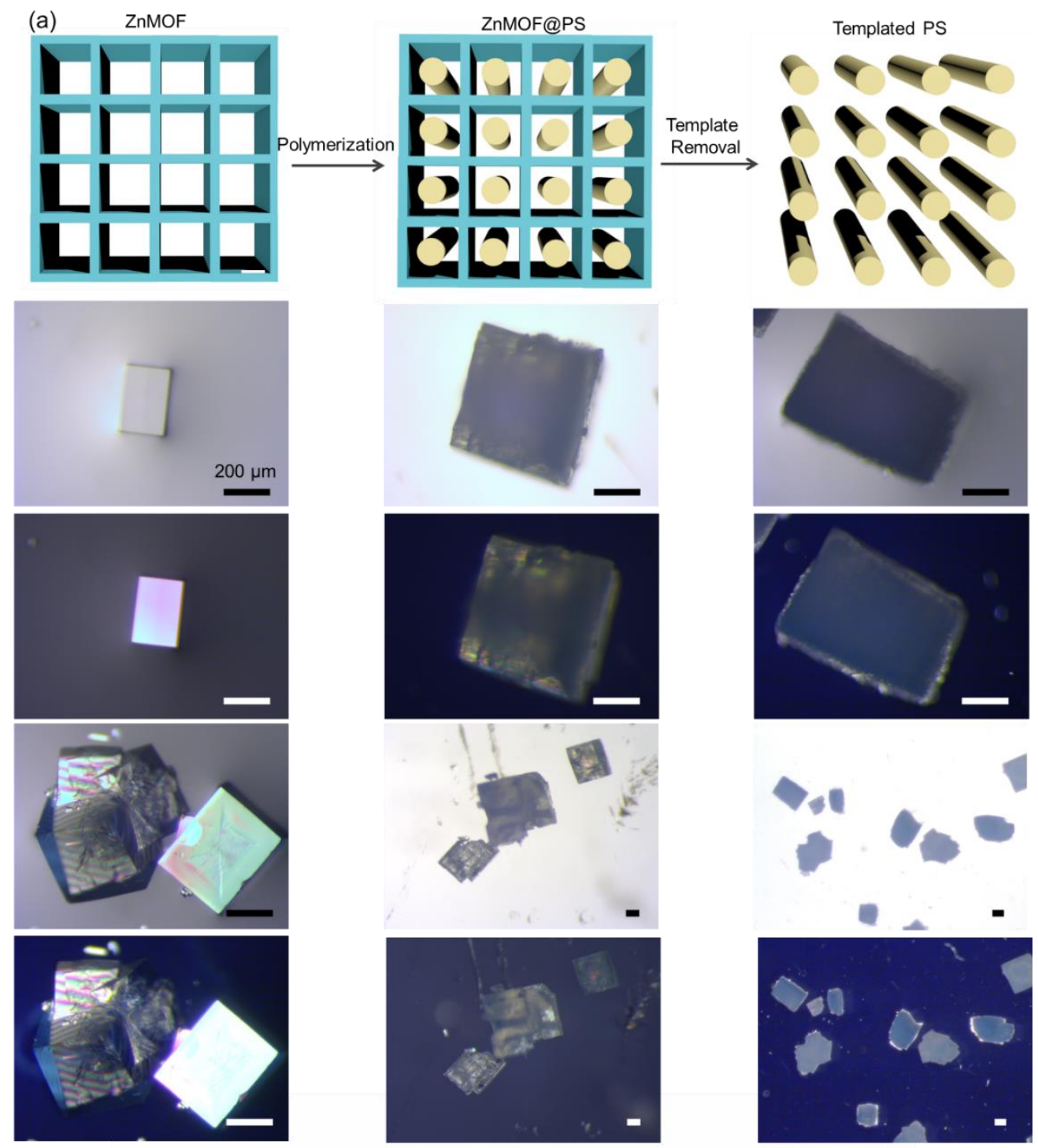

(b)

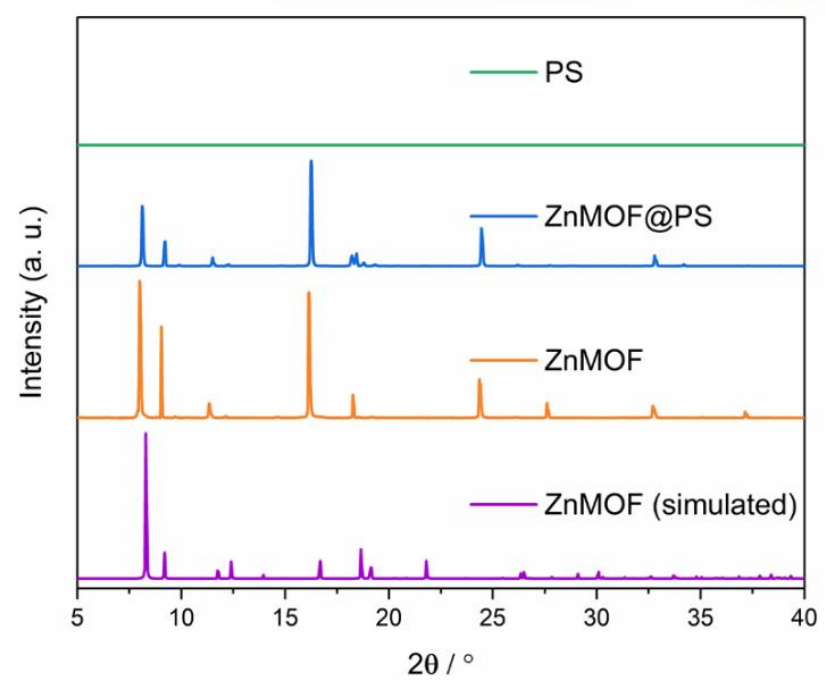

Figure S2. (a) Models and optical images showing the stepwise polymerization and template removal processes in $\mathrm{Zn}_{2}(\mathrm{BDC})_{2}$ (TED). (b) PXRD patterns of $\mathrm{Zn}_{2}(\mathrm{BDC})_{2}(\mathrm{TED}), \mathrm{Zn}_{2}(\mathrm{BDC})_{2}(\mathrm{TED}) @ \mathrm{PS}$ and templated PS. (Cyan frameworks = $\mathrm{Zn}_{2}(\mathrm{BDC})_{2}(\mathrm{TED}) ;$ yellow rods $\left.=\mathrm{PS}\right)$ 

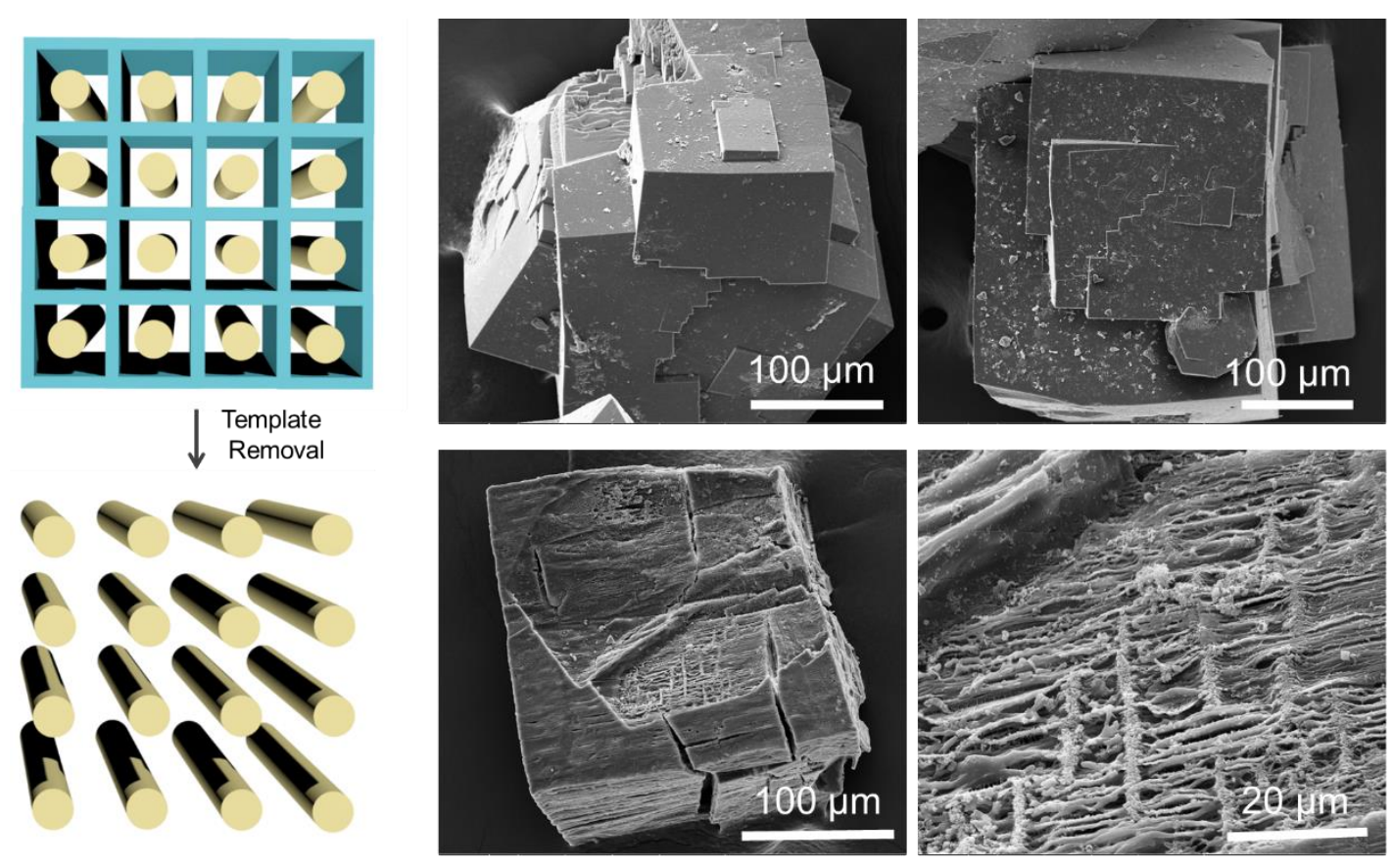

Figure S3. Models and SEM images showing the cubic feature of $\mathrm{Zn}_{2}(\mathrm{BDC})_{2}$ (TED)@PS and the maintained cubic feature of PS after template removal. $\left(\right.$ Cyan frameworks $=\mathrm{Zn}_{2}(\mathrm{BDC})_{2}(\mathrm{TED})$; yellow rods $\left.=\mathrm{PS}\right)$

Synthesis of PCN-222 nanorods. Nano-sized PCN-222 rods were synthesized on the basis of previous reports with slight modifications. ${ }^{[2]} \mathrm{ZrOCl}_{2} \bullet 8 \mathrm{H}_{2} \mathrm{O}(38 \mathrm{mg})$, TCPP (tetrakis(4-carboxyphenyl)porphyrin, $6.5 \mathrm{mg}$ ) and dichloroacetic acid $(0.25 \mathrm{~mL})$ in DMF (6 mL) were ultrasonically dissolved in a $20 \mathrm{~mL}$ Pyrex vial. The mixture was heated at $130^{\circ} \mathrm{C}$ in an oven for $2 \mathrm{~h}$. After the mixture was cooled to room temperature, the resulted purple nanorods were collected by centrifugation, followed by solvent exchange with DMF for three times.

Synthesis of PCN-222 microneedles. PCN-222 microneedles were synthesized on the basis of previous reports with slight modifications. ${ }^{[3]} \mathrm{ZrCl}_{4}$ (70 mg), TCPP (50 mg) and benzoic acid $(2700 \mathrm{mg}$ ) in DEF (N,N-diethylformamide, $8 \mathrm{~mL}$ ) were ultrasonically dissolved in a $20 \mathrm{~mL}$ Pyrex vial. The mixture was heated at $120{ }^{\circ} \mathrm{C}$ in an oven for $48 \mathrm{~h}$. After the mixture was cooled to room temperature, needle-shaped microcrystals were harvested by filtration. The resulted crystals were washed three times with DMF and three times with acetone, respectively. The solvent was removed under vacuum at $100{ }^{\circ} \mathrm{C}$ for $24 \mathrm{~h}$.

Synthesis of PCN-222 large crystals. Large PCN-222 crystals were synthesized on the basis of previous reports with slight modifications. ${ }^{[4]} \mathrm{ZrOCl}_{2} \bullet 8 \mathrm{H}_{2} \mathrm{O}(47 \mathrm{mg})$, TCPP 
$(25 \mathrm{mg})$ and 4-tert-butylbenzoic acid $(2700 \mathrm{mg})$ in DEF $(6 \mathrm{~mL})$ were ultrasonically dissolved in a $20 \mathrm{~mL}$ Pyrex vial. The mixture was heated at $120^{\circ} \mathrm{C}$ in an oven for $12 \mathrm{~h}$. After the mixture was cooled to room temperature, large needle crystals were harvested by filtration. The resulted crystals were washed three times with DMF and three times with acetone, respectively. The solvent was removed under vacuum at $100{ }^{\circ} \mathrm{C}$ for $24 \mathrm{~h}$.

Synthesis of PCN-222@Znz(BDC)2(TED). The hierarchical MOF-on-MOF composites were synthesized on the basis of previous reports with slight modifications. ${ }^{[5]}$ Activated PCN-222 (0.012 g) was immersed in a solution of BDC $(0.084 \mathrm{~g})$ in $3 \mathrm{~mL}$ DMF in a Pyrex vial. The mixture was heated in a $100{ }^{\circ} \mathrm{C}$ oven for 6 h. A solution of TED $(0.028 \mathrm{~g})$ and $\mathrm{Zn}\left(\mathrm{NO}_{3}\right)_{2} \cdot 9 \mathrm{H}_{2} \mathrm{O}(0.150 \mathrm{~g})$ in $3 \mathrm{~mL}$ DMF were further added into the vial. The mixture was heated in a $100{ }^{\circ} \mathrm{C}$ oven for 2 days to generate the crystals of PCN-222@ $\mathrm{Zn}_{2}(\mathrm{BDC})_{2}$ (TED). The solution was decanted, and the crystals were repeatedly washed with DMF. The ratio of PCN-222 and $\mathrm{Zn}_{2}(\mathrm{BDC})_{2}(\mathrm{TED})$ in the hierarchical MOF-on-MOF architectures can be easily controlled by tuning the feed ratios. The tunable sizes of PCN-222 in the hierarchical MOF-on-MOF architectures can also be achieved.

\section{Polymerization of vinyl monomers in hierarchical MOFs followed by template}

removal. The dried host compound MOFs@ $\mathrm{Zn}_{2}(\mathrm{BDC})_{2}$ (TED) (100 mg)was prepared by evacuation $(<0.1 \mathrm{kPa})$ at $130{ }^{\circ} \mathrm{C}$ for 1 day in a Pyrex reaction tube. Subsequently, $\mathrm{Zn}_{2}(\mathrm{BDC})_{2}$ (TED) was immersed in a purified monomer $(0.5 \mathrm{~mL})$ with AIBN $(3 \mathrm{mg})$ at room temperature for $0.5 \mathrm{~h}$ to incorporate the monomer and the radical initiator into the nanochannels. After excess monomer external to the host crystals was removed completely by evacuation (St: $0.2 \mathrm{kPa}, \mathrm{MMA}: 2.0 \mathrm{kPa}$ ) at room temperature for $0.5 \mathrm{~h}$, the reaction tube was filled with nitrogen, and heated to $70{ }^{\circ} \mathrm{C}$ to perform the polymerization of St and MMA for 48 and $24 \mathrm{~h}$, respectively. To isolate the polymer inside the channels, the composite was stirred for 4 days in a $0.05 \mathrm{M}$ aqueous solution $(40 \mathrm{~mL})$ of sodium ethylenediaminetetraacetate (Na-EDTA) for the complete dissolution of the frameworks of $\mathrm{Zn}_{2}$ (BDC) $)_{2}$ (TED). The collected hierarchical MOFon-polymer composites were washed with water and dried under a reduced pressure at room temperature. 
(a)
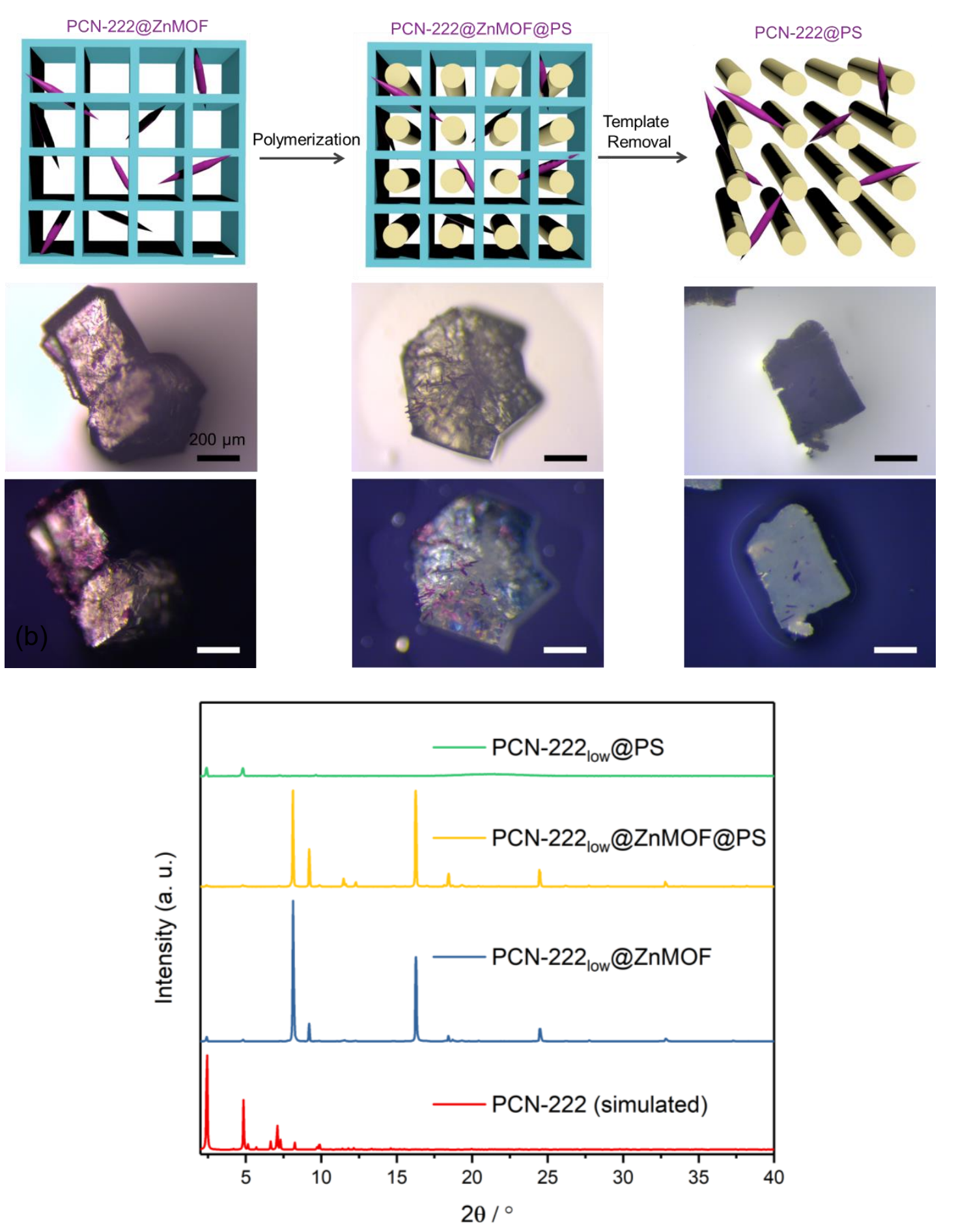

Figure S4. (a) Models and optical images showing the stepwise polymerization and template removal processes in hierarchical PCN-222@ $\mathrm{Zn}_{2}(\mathrm{BDC})_{2}(\mathrm{TED})$ with low concentration of PCN-222. (b) PXRD patterns of PCN-222@ $\mathrm{Zn}_{2}(\mathrm{BDC})_{2}$ (TED), PCN222@Zn 2 (BDC)2(TED)@PS and PCN-222@PS with low concentrations of PCN-222. $\left(\right.$ Cyan frameworks $=\mathrm{Zn}_{2}(\mathrm{BDC})_{2}(\mathrm{TED})$; yellow rods $=\mathrm{PS}$; purple needles $\left.=\mathrm{PCN}-222\right)$ 
(a)PCN-222@ZnMOF
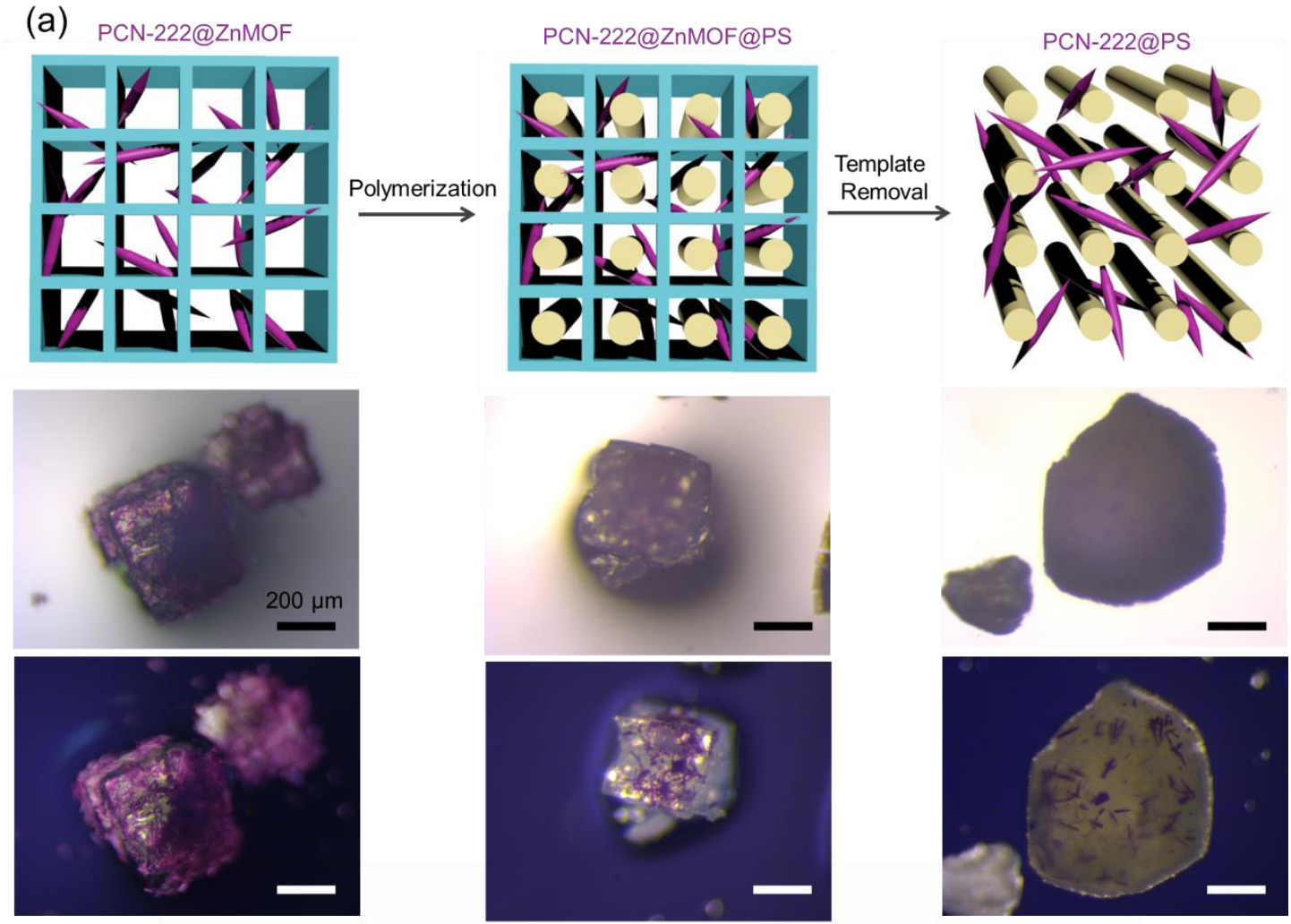

(b)

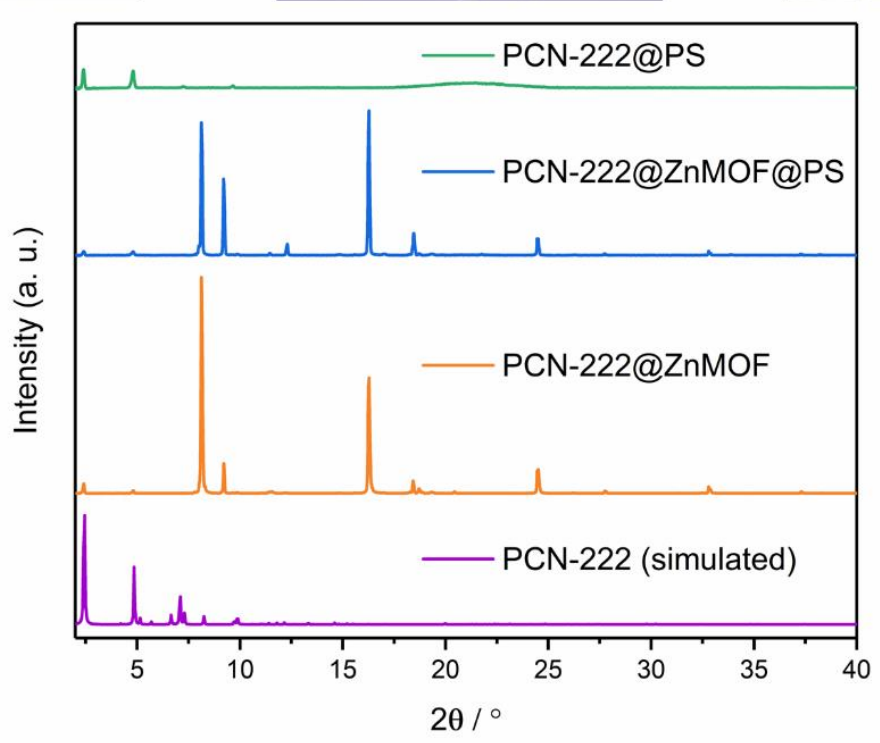

Figure S5. (a) Models and optical images showing the stepwise polymerization and template removal processes in hierarchical PCN-222@ $\mathrm{Zn}_{2}(\mathrm{BDC})_{2}(\mathrm{TED})$ with moderate concentration of PCN-222. (b) PXRD patterns of PCN-

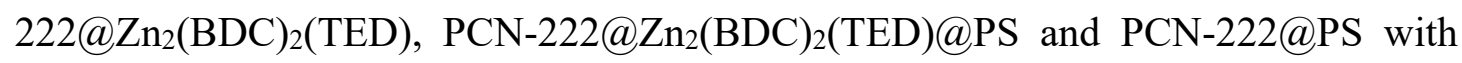
moderate concentrations of PCN-222. (Cyan frameworks $=\mathrm{Zn}_{2}(\mathrm{BDC})_{2}(\mathrm{TED})$; yellow rods $=$ PS; purple needles $=$ PCN-222) 
(a)

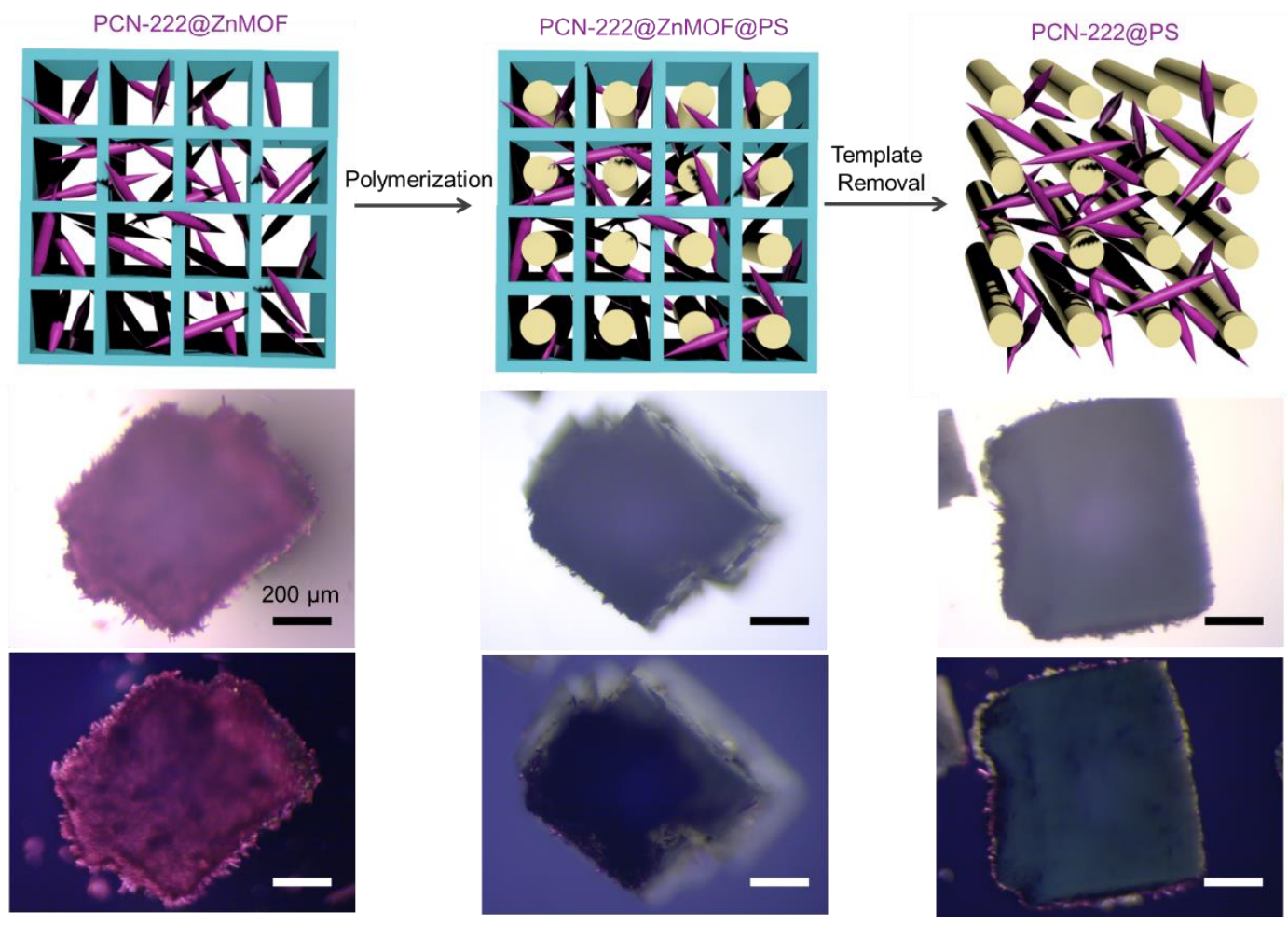

(b)

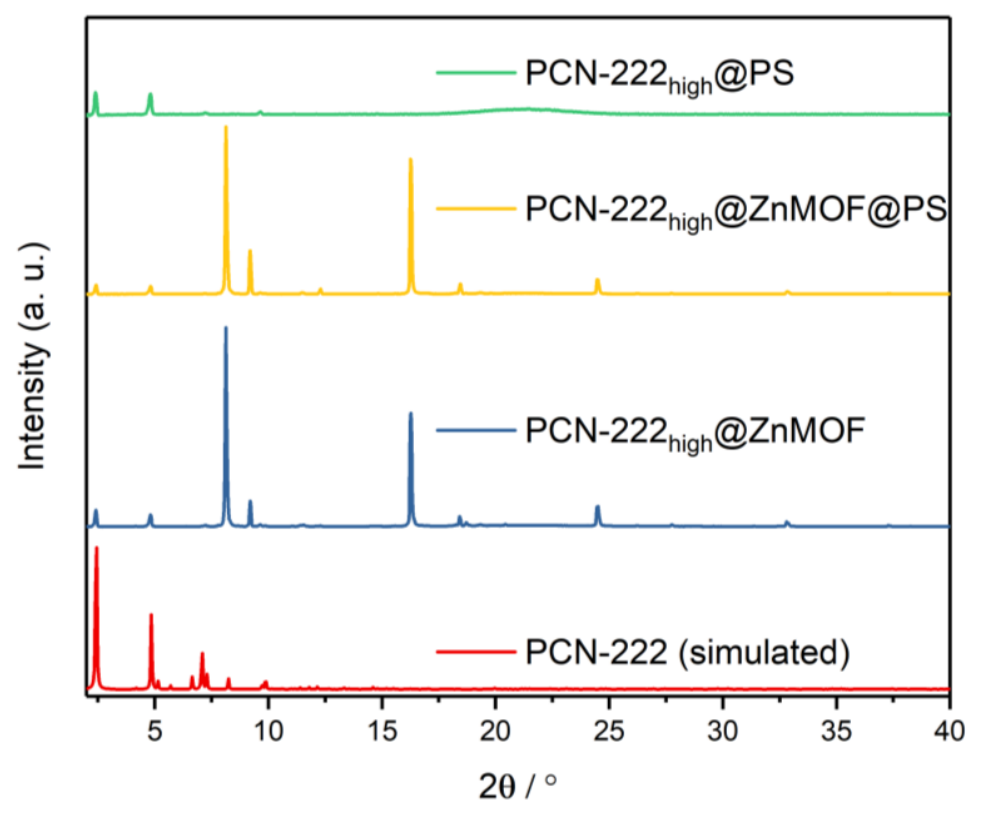

Figure S6. (a) Models and optical images showing the stepwise polymerization and template removal processes in hierarchical PCN-222@ $\mathrm{Zn}_{2}(\mathrm{BDC})_{2}(\mathrm{TED})$ with high concentration of PCN-222. (b) PXRD patterns of PCN-222@Zn2(BDC)2(TED), PCN222@Zn2(BDC)2(TED)@PS and PCN-222@PS with high concentrations of PCN-222. $\left(\right.$ Cyan frameworks $=\mathrm{Zn}_{2}(\mathrm{BDC})_{2}(\mathrm{TED})$; yellow rods $=\mathrm{PS}$; purple needles $\left.=\mathrm{PCN}-222\right)$ 
(a)

PCN-222@ZnMOF
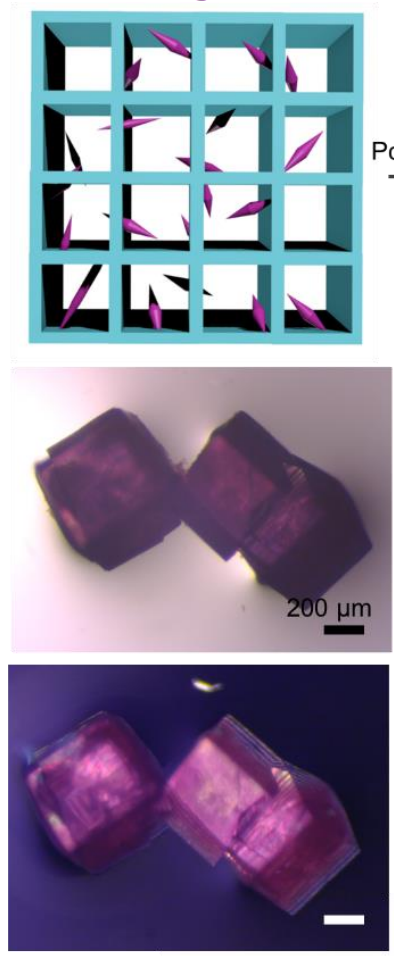

PCN-222@ZnMOF@PS
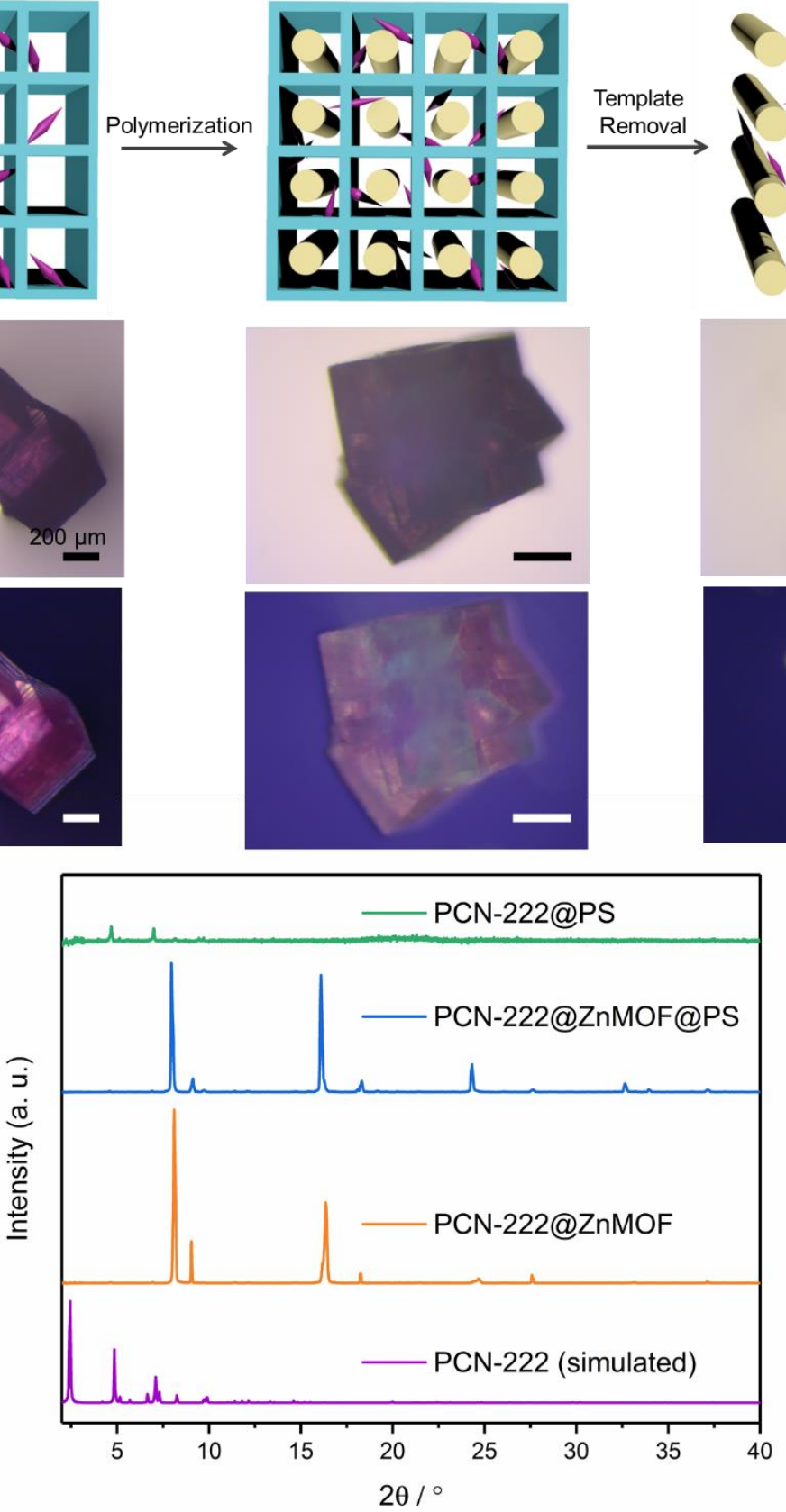

PCN-222@PS
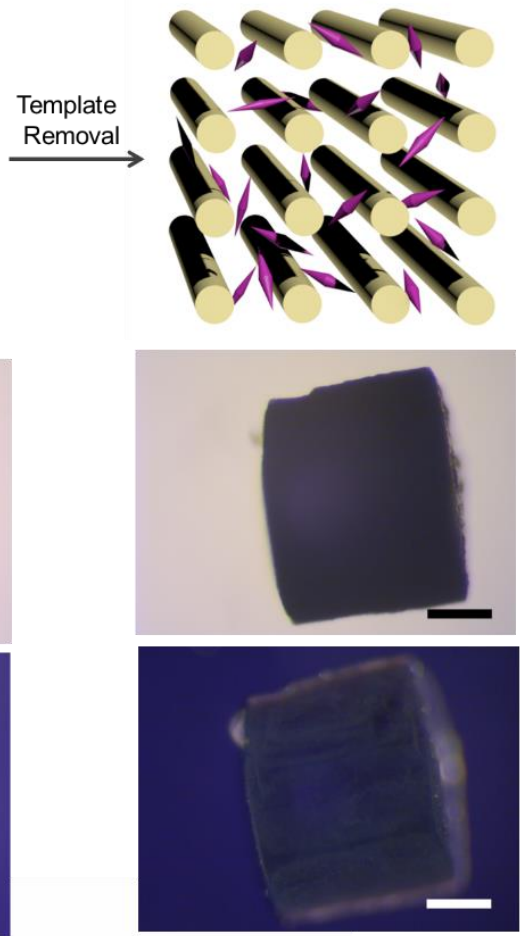

(b)

Figure S7. (a) Models and optical images showing the stepwise polymerization and template removal processes in hierarchical PCN-222 nanorods@ $\mathrm{Zn}_{2}(\mathrm{BDC})_{2}(\mathrm{TED})$. (b) PXRD patterns of PCN-222 nanorods@ $Z_{2}(\mathrm{BDC})_{2}$ (TED), $\quad$ PCN-222 nanorods@Zn 2 (BDC)2 $(\mathrm{TED}) @ \mathrm{PS}$ and PCN-222 nanorods@PS. (Cyan frameworks = $\mathrm{Zn}_{2}(\mathrm{BDC})_{2}(\mathrm{TED}) ;$ yellow rods $=\mathrm{PS}$; purple needles $\left.=\mathrm{PCN}-222\right)$ 
(a)

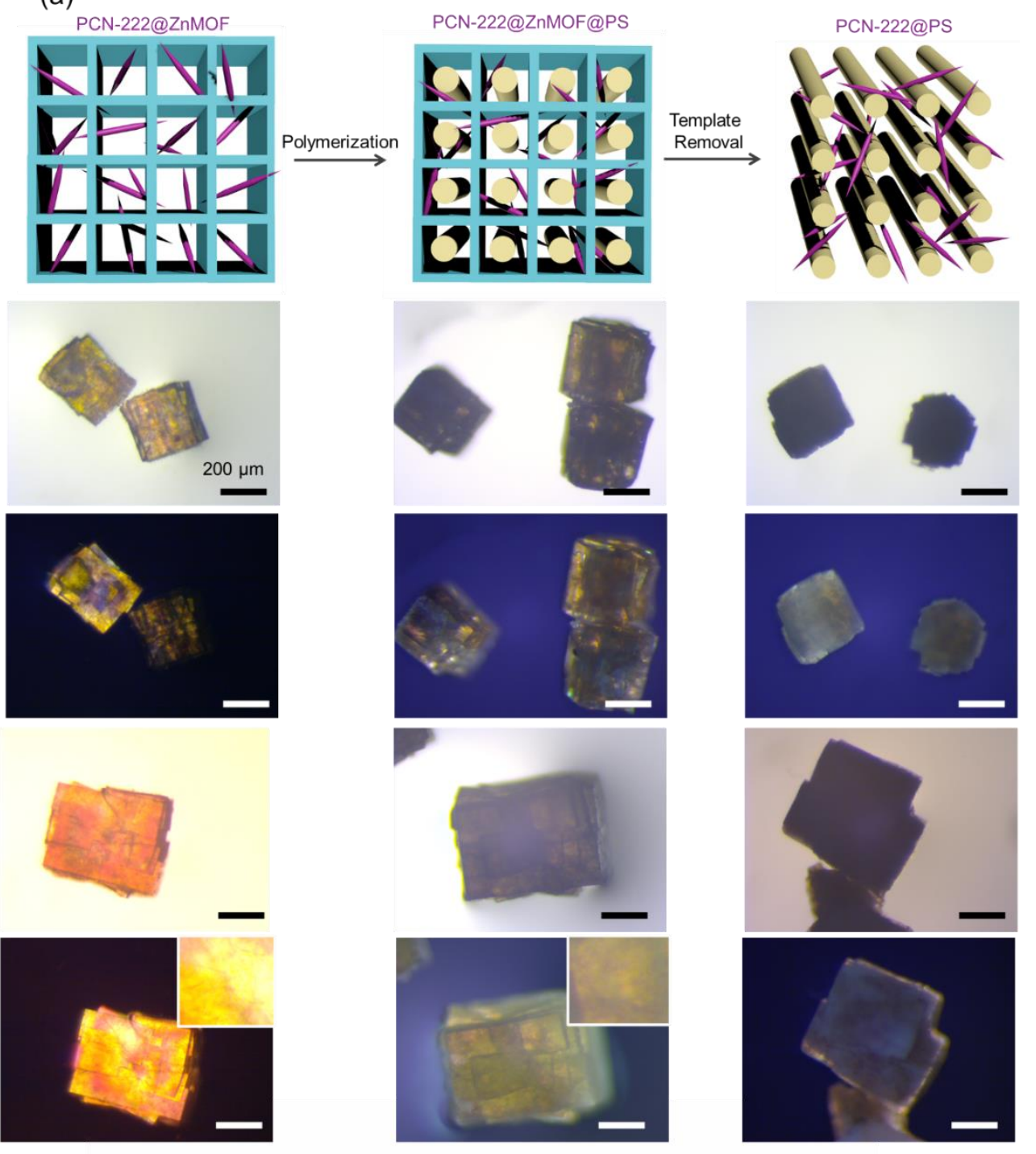

(b)

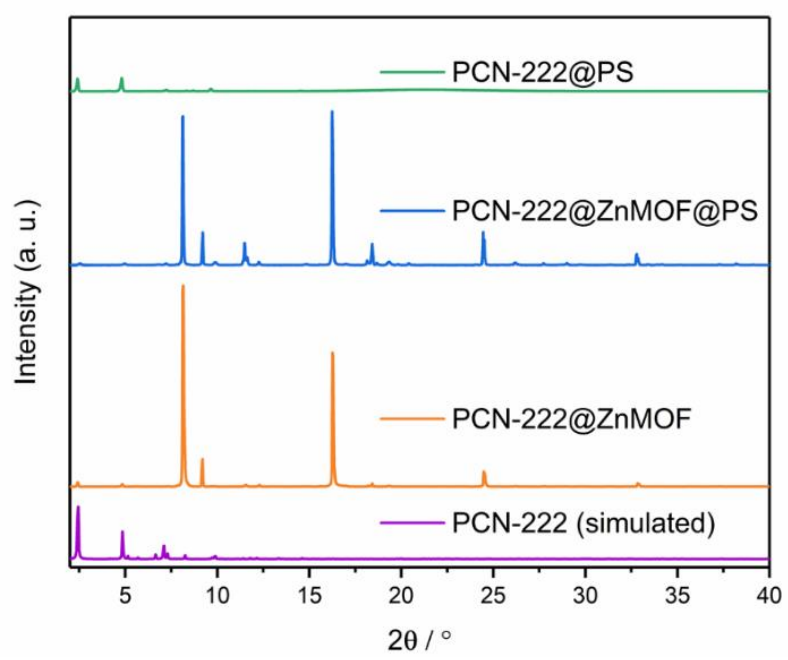

Figure S8. (a) Models and optical images showing the stepwise polymerization and template removal processes in hierarchical PCN-222 microneedles@ $\mathrm{Zn}_{2}(\mathrm{BDC})_{2}(\mathrm{TED})$. (b) PXRD patterns of PCN-222 microneedles@ $\mathrm{Zn}_{2}(\mathrm{BDC})_{2}$ (TED), PCN-222 microneedles@Zn 2 (BDC)2(TED)@PS and PCN-222 microneedles@PS. (Cyan frameworks $=\mathrm{Zn}_{2}(\mathrm{BDC})_{2}(\mathrm{TED}) ;$ yellow rods $=\mathrm{PS} ;$ purple needles $\left.=\mathrm{PCN}-222\right)$ 
(a) PCN-222 Nanorods in Hierarchical Architectures
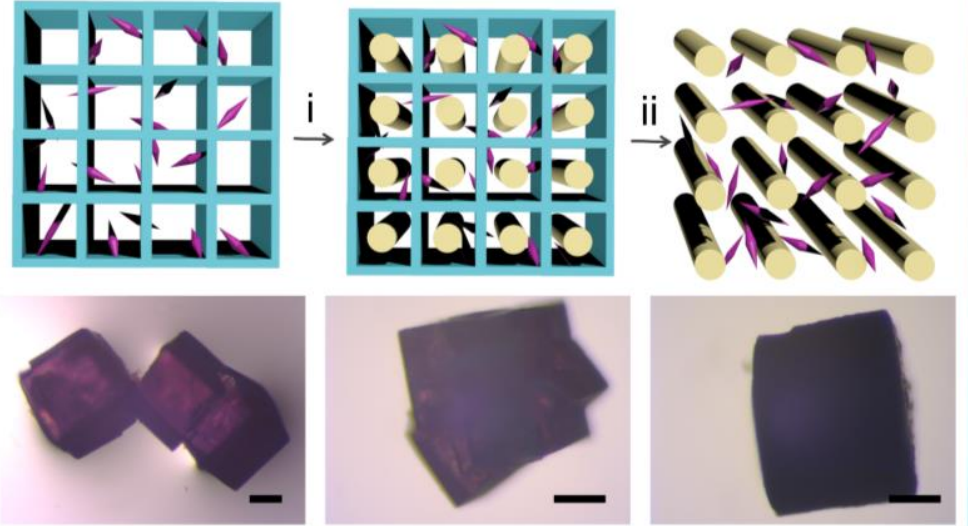

(b) PCN-222 Microneedles in Hierarchical Architectures
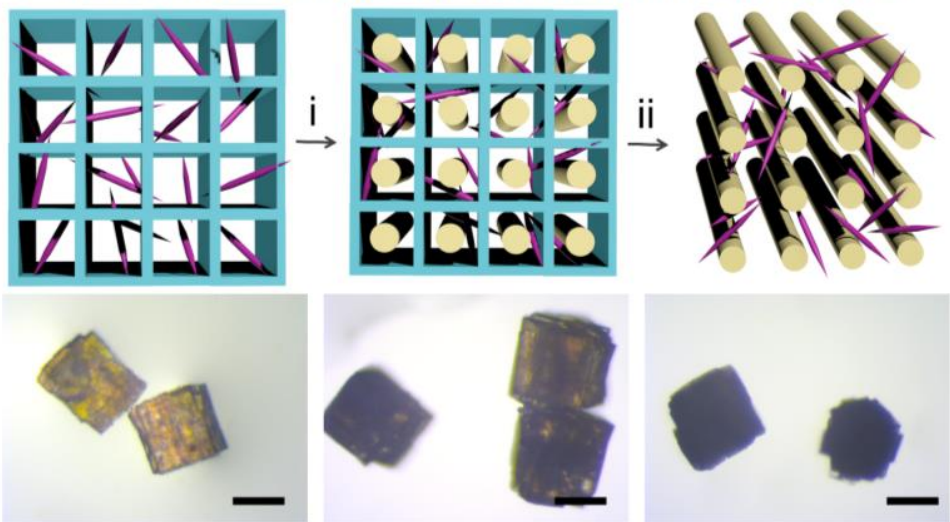

(c)

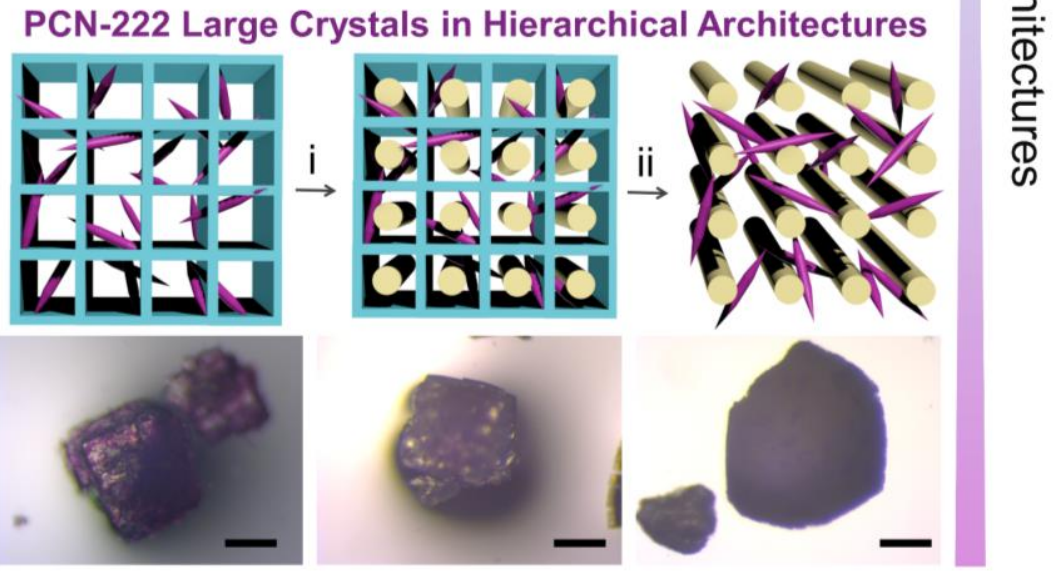

Figure S9. Tunable sizes of PCN-222 in hierarchical MOF-on-MOF and MOF-onpolymer architectures. Models and optical images showing the immobilization of (a) PCN-222 nanorods, (b) PCN-222 microneedles and (c) PCN-222 large crystals in MOF-on-MOF and MOF-on-polymer composites. Scale bar is $200 \mu \mathrm{m}$. (Cyan frameworks $=\mathrm{Zn}_{2}(\mathrm{BDC})_{2}(\mathrm{TED}) ;$ yellow rods $=\mathrm{PS} ;$ purple needles $\left.=\mathrm{PCN}-222\right)$ 

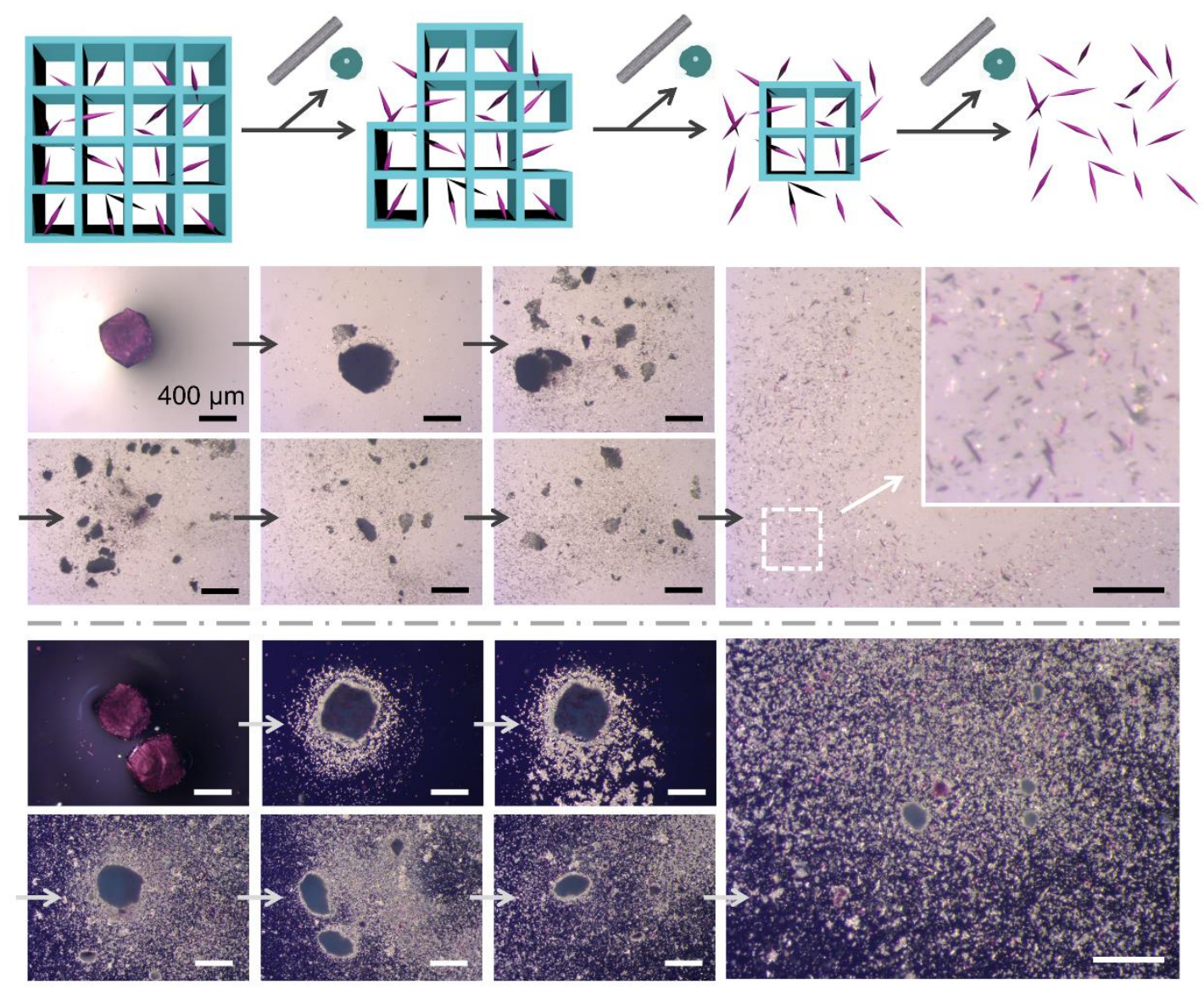

Figure S10. Illustration of the decomposition of $\mathrm{Zn}_{2}(\mathrm{BDC})_{2}(\mathrm{TED})$ and preservation of PCN-222 under Na-EDTA treatment. Optical images of PCN-222@ $Z_{2}(B D C)_{2}(T E D)$ crystals treated with Na-EDTA solutions shown fast decomposition of $\mathrm{Zn}_{2}$ (BDC) 2 (TED) framework when in the absence of polymerization, while stable PCN-222 survived after Na-EDTA treatment. $\left(\right.$ Cyan frameworks $=\mathrm{Zn}_{2}(\mathrm{BDC})_{2}(\mathrm{TED})$; yellow rods $=\mathrm{PS}$; purple needles $=$ PCN-222) 


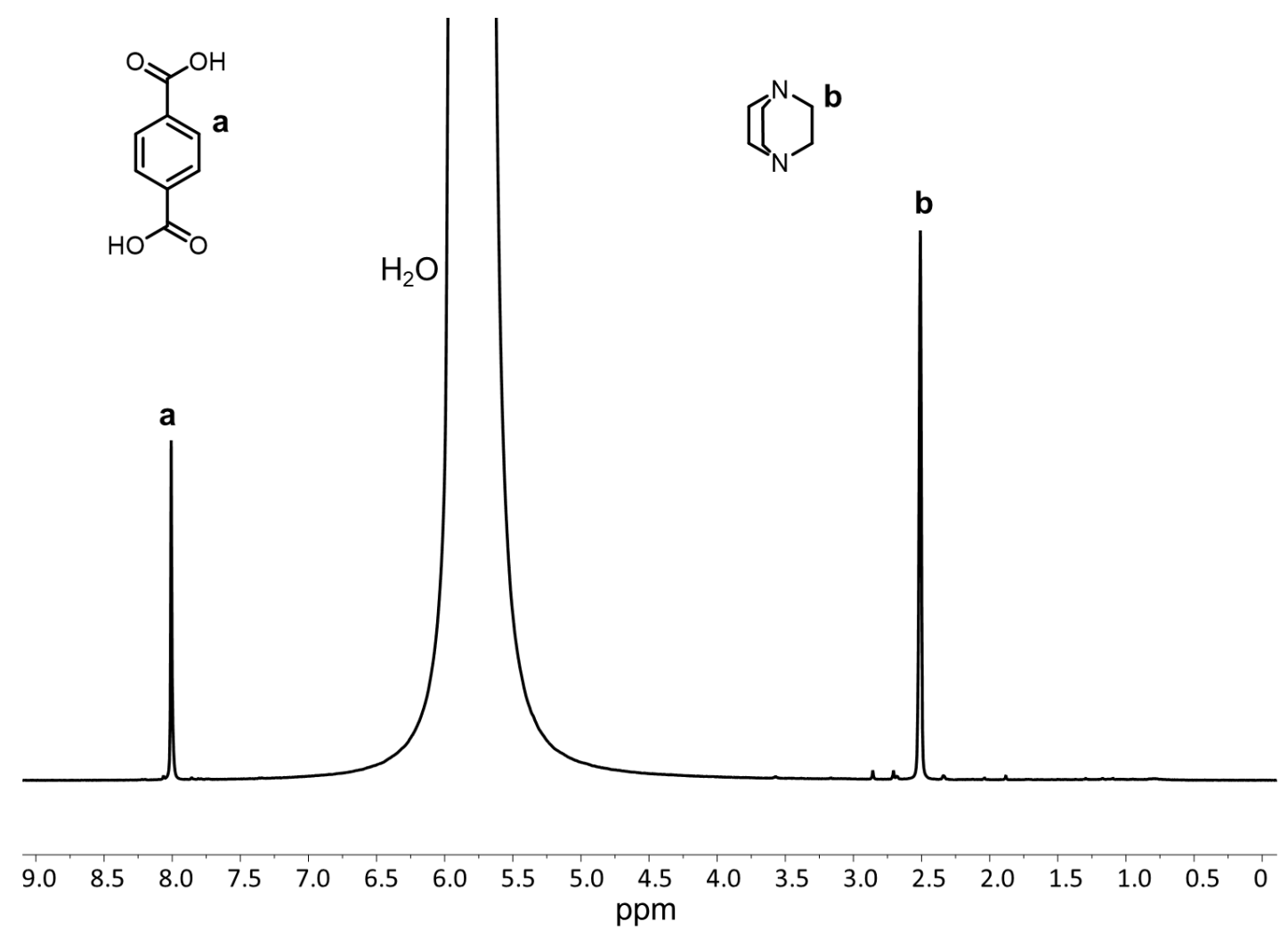

Figure S11. ${ }^{1} \mathrm{H}$ NMR spectroscopy of digested $\mathrm{Zn}_{2}(\mathrm{BDC})_{2}$ (TED) in $\mathrm{d}_{6}$-DMSO.

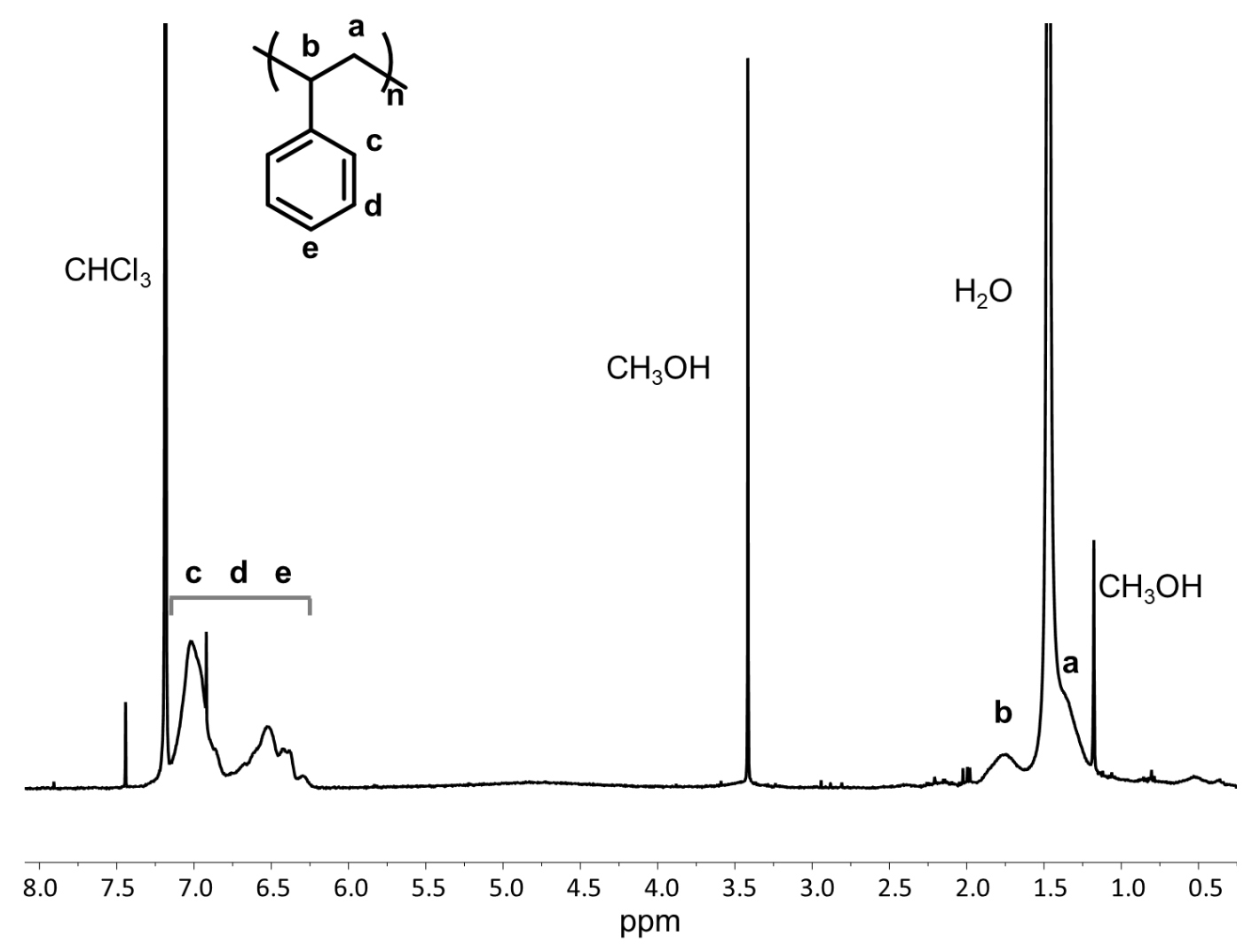

Figure S12. ${ }^{1} \mathrm{H}$ NMR spectroscopy of templated PS in $\mathrm{CDCl}_{3}$. 

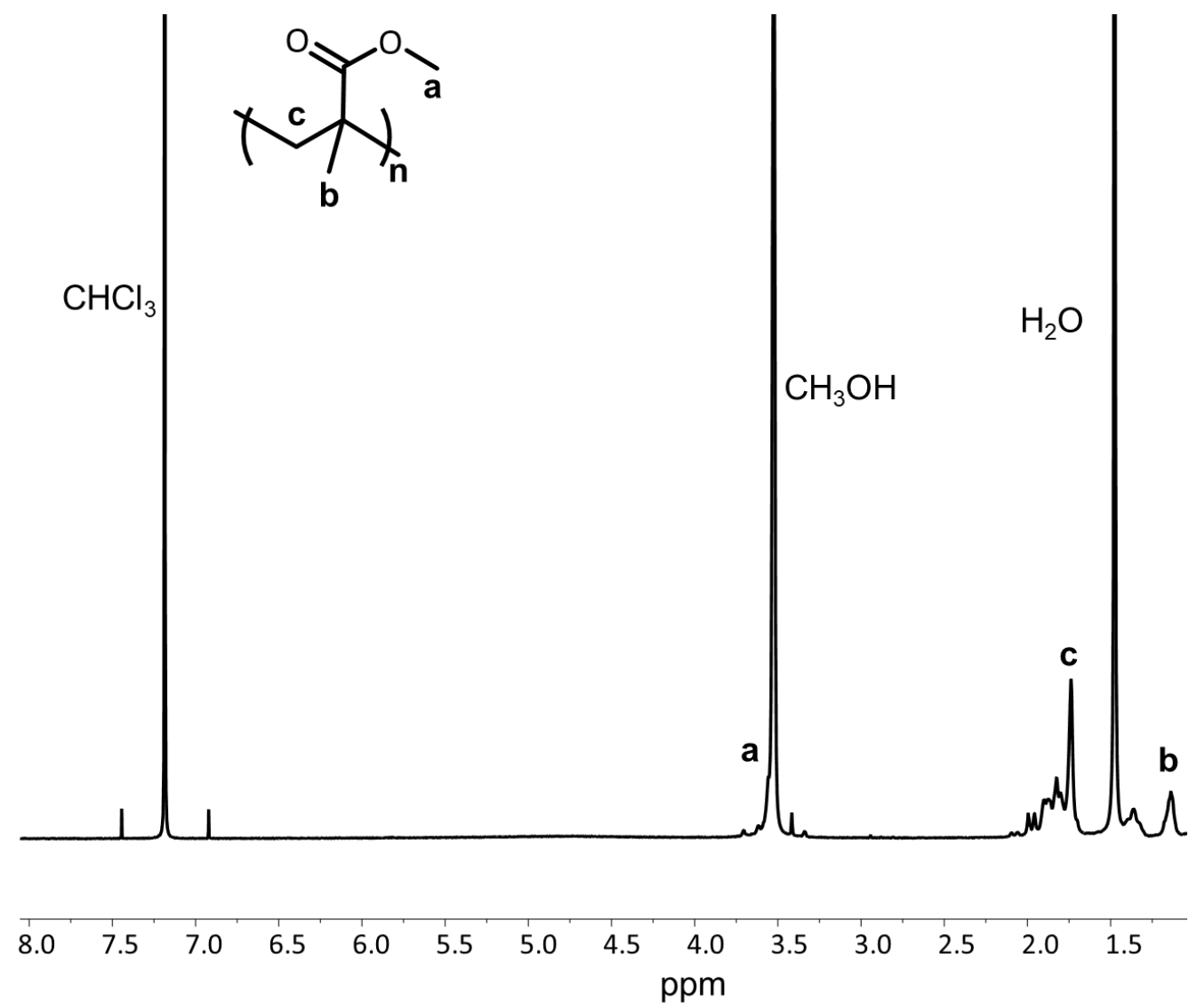

Figure S13. ${ }^{1} \mathrm{H}$ NMR spectroscopy of templated PMMA in $\mathrm{CDCl}_{3}$. 


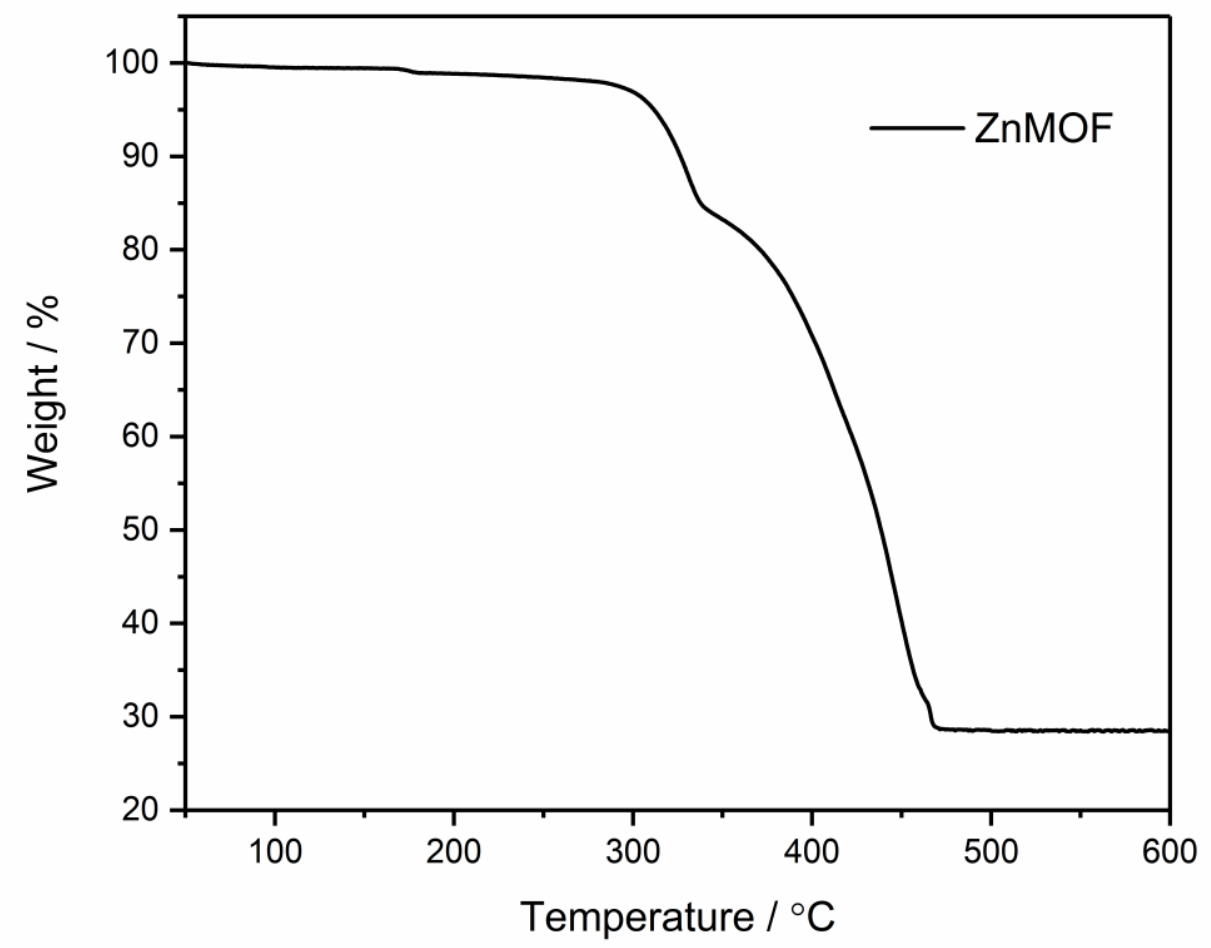

Figure S14. Thermogravimetric analysis (TGA) of $\mathrm{Zn}_{2}(\mathrm{BDC})_{2}$ (TED).

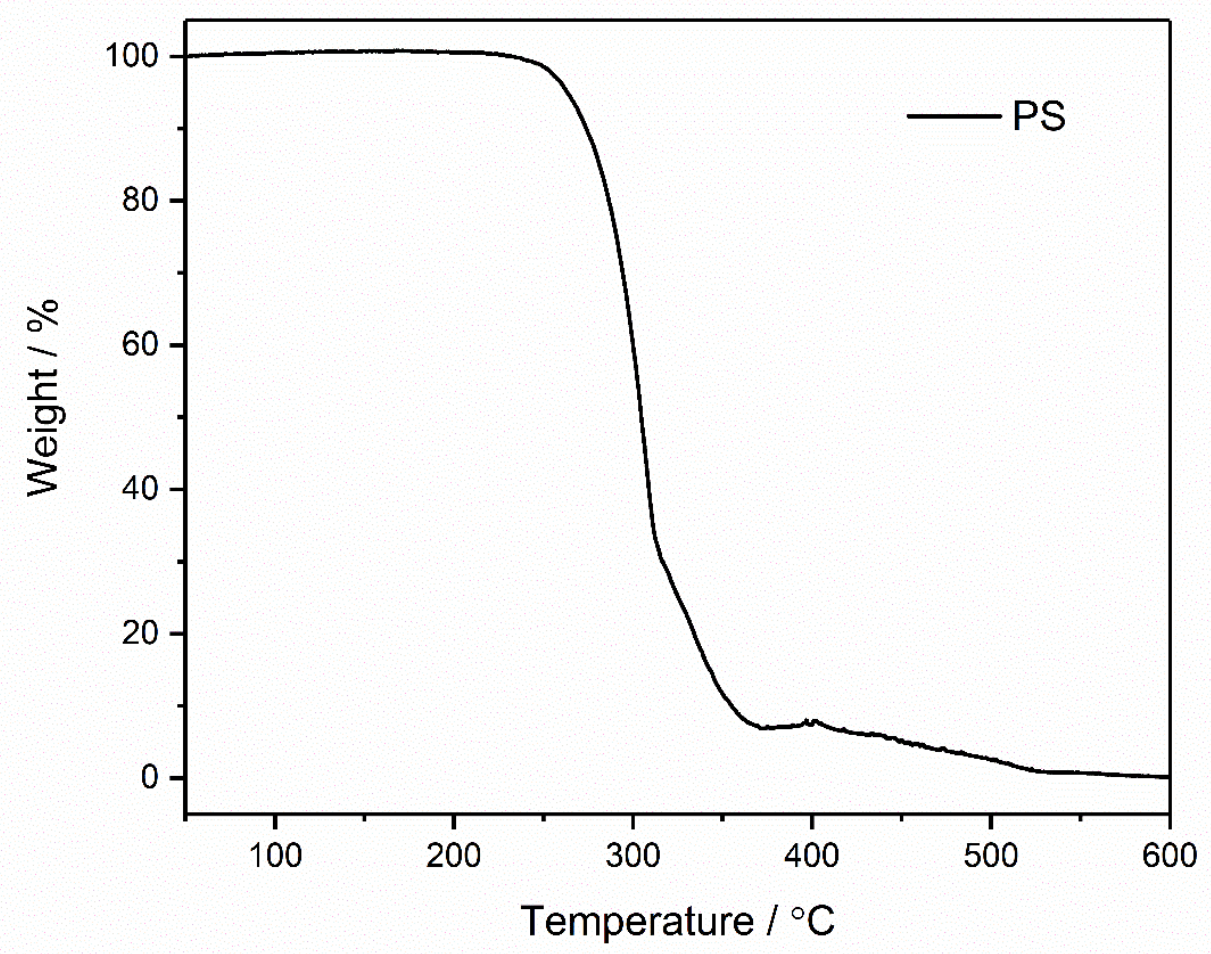

Figure S15. Thermogravimetric analysis (TGA) of templated PS. 


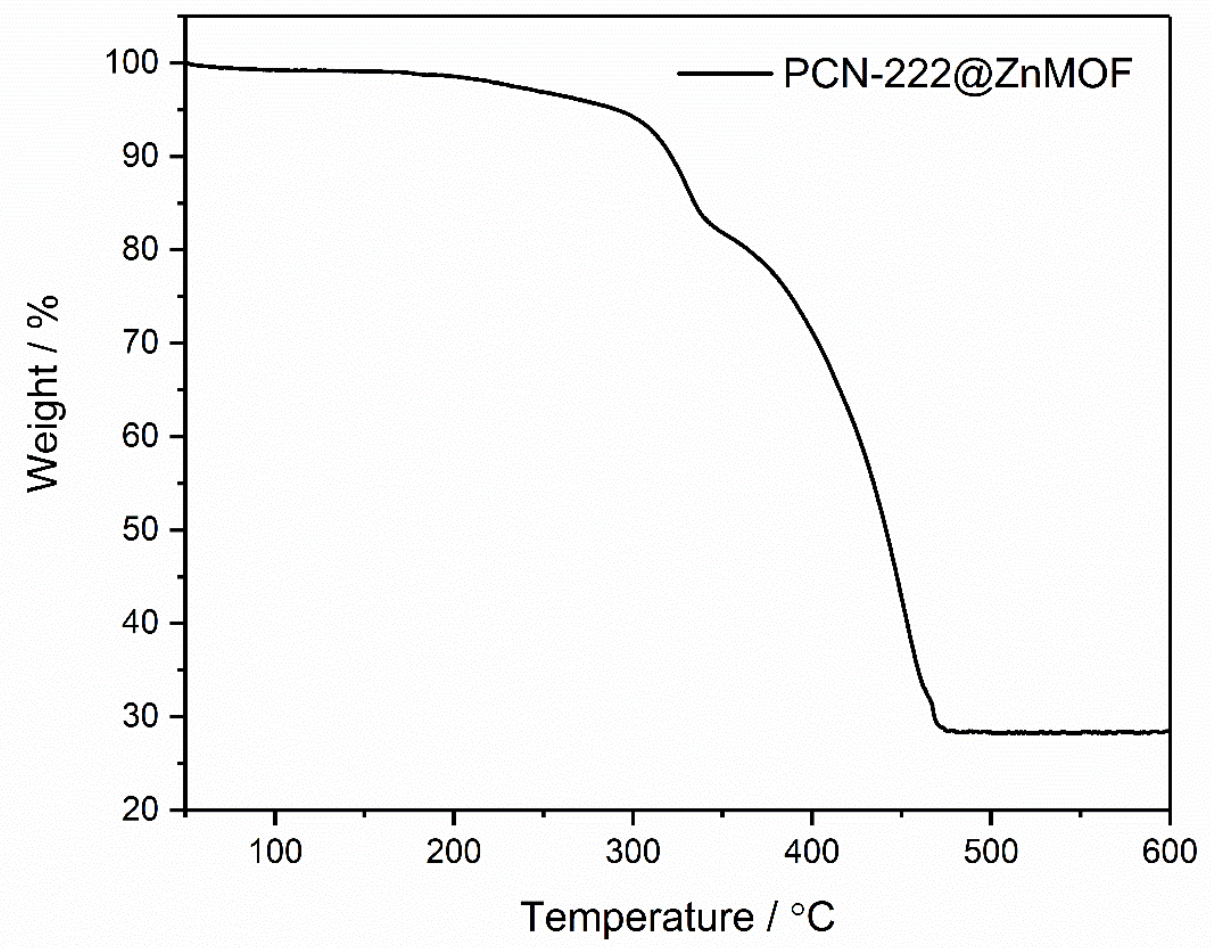

Figure S16. Thermogravimetric analysis (TGA) of PCN-222@ $\mathrm{Zn}_{2}(\mathrm{BDC})_{2}(\mathrm{TED})$.

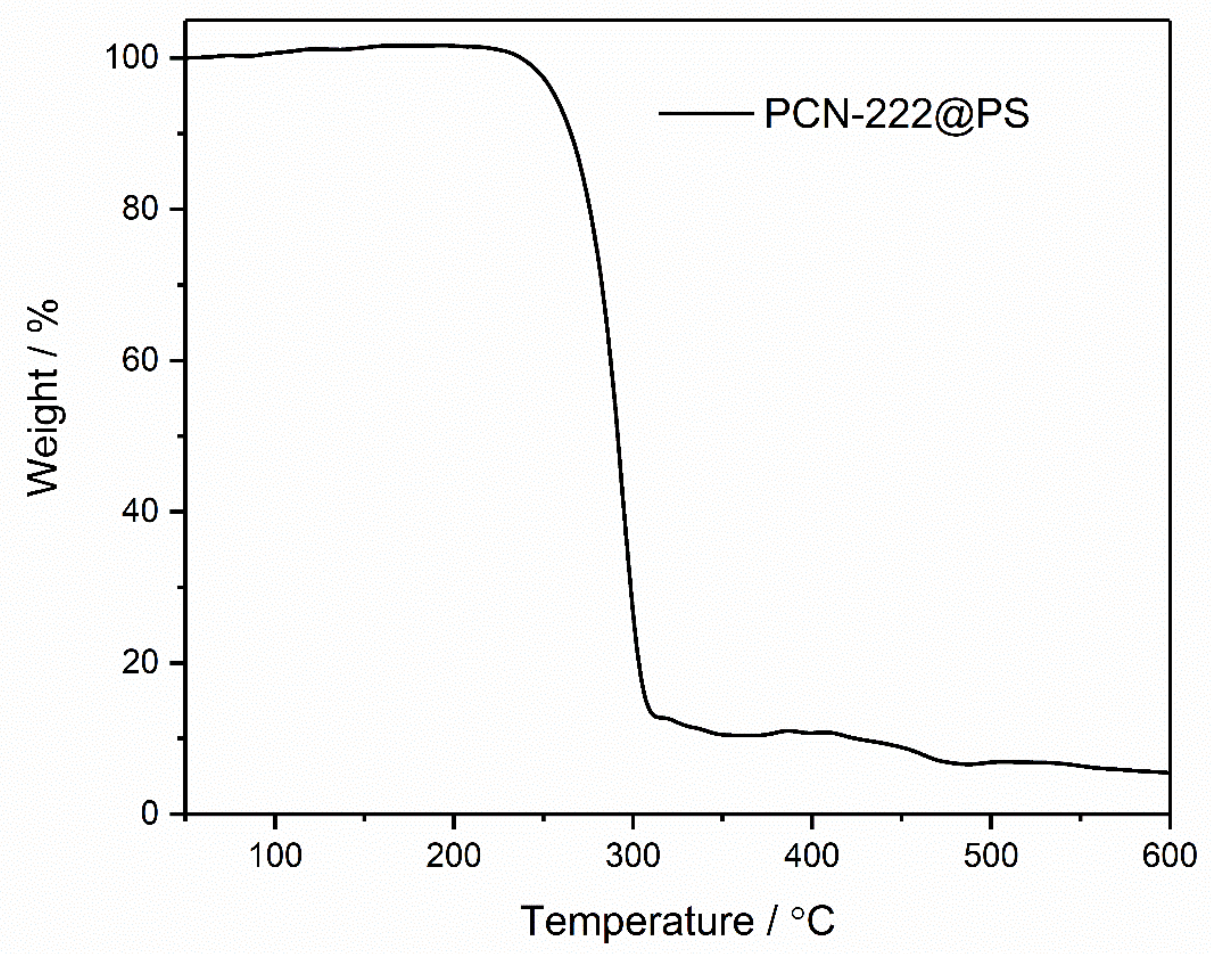

Figure S17. Thermogravimetric analysis (TGA) of templated PCN-222@PS. 


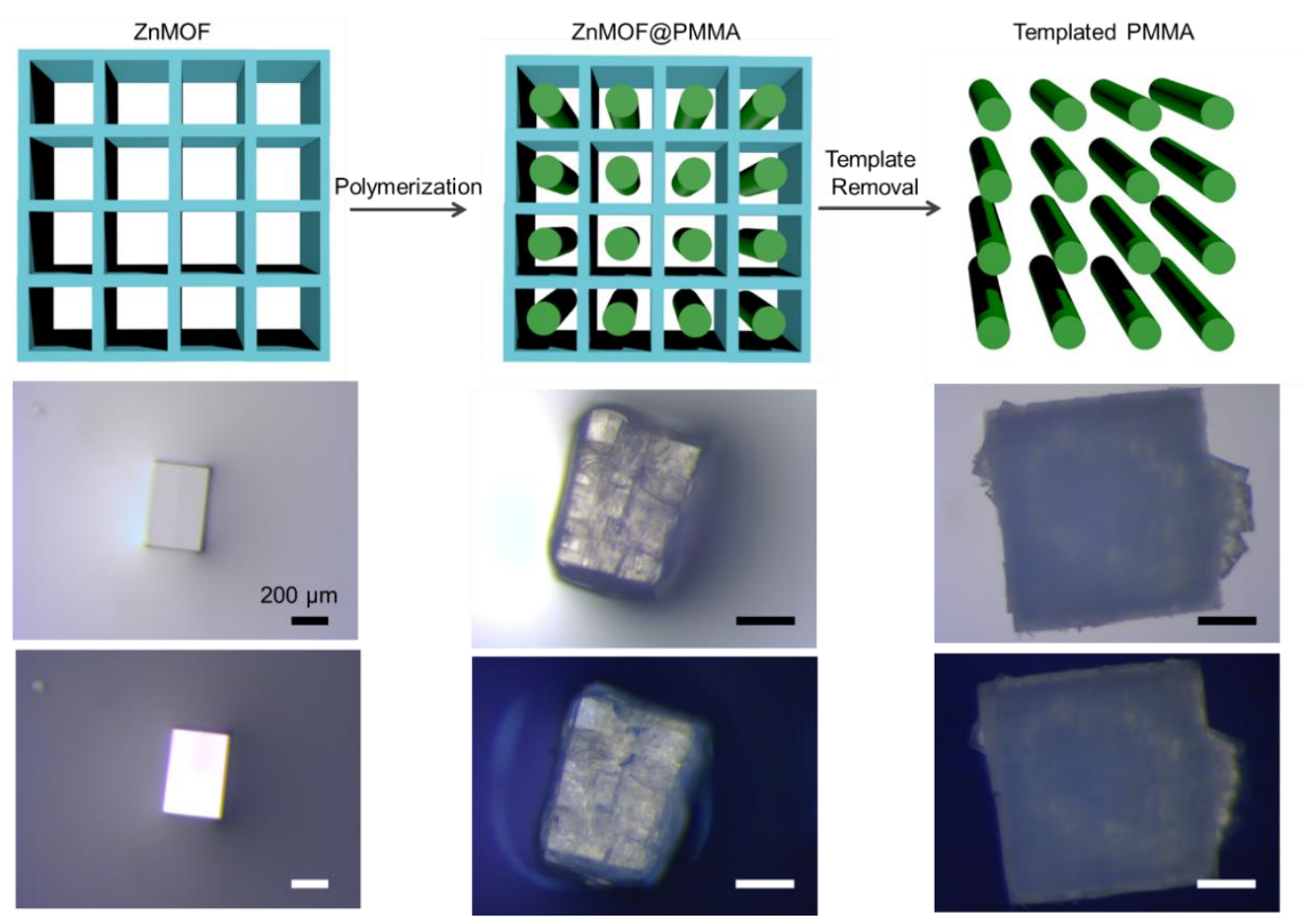

Figure S18. Models and optical images showing the stepwise polymerization and template removal processes from $\mathrm{Zn}_{2}$ (BDC) $)_{2}$ (TED) to $\mathrm{Zn}_{2}(\mathrm{BDC})_{2}$ (TED)@PMMA, to templated PMMA. $\left(\right.$ Cyan frameworks $=\mathrm{Zn}_{2}(\mathrm{BDC})_{2}(\mathrm{TED})$; green rods $\left.=\mathrm{PMMA}\right)$

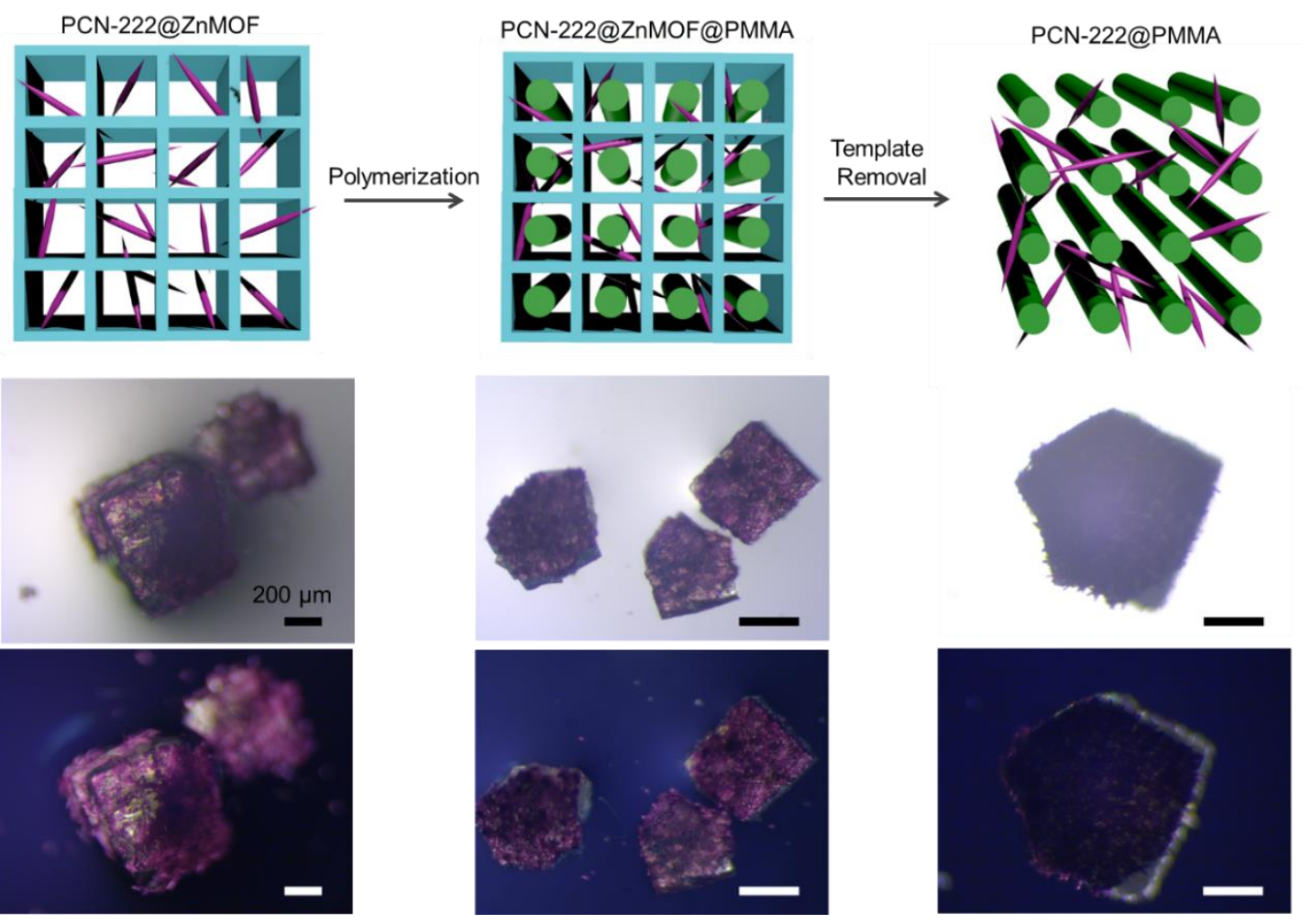

Figure S19. Models and optical images showing the stepwise polymerization and template removal processes from hierarchical PCN-222@ $\mathrm{Zn}_{2}(\mathrm{BDC})_{2}(\mathrm{TED})$ to PCN222@Zn 2 (BDC)2(TED)@PMMA, to PCN-222@PMMA. (Cyan frameworks = 
$\mathrm{Zn}_{2}(\mathrm{BDC})_{2}(\mathrm{TED})$; green rods $=$ PMMA; purple needles $\left.=\mathrm{PCN}-222\right)$

Synthesis of PCN-160. PCN-160 was synthesized according to the literature with slight modification. ${ }^{[6]} \mathrm{ZrCl}_{4}(200 \mathrm{mg}), \mathrm{H}_{2} \mathrm{AZDC}(100 \mathrm{mg})$, trifluoroacetic acid $(1.0 \mathrm{~mL})$ and DMF $(20 \mathrm{~mL})$ were charged in a Pyrex vial. The mixture was heated in $120^{\circ} \mathrm{C}$ oven for $72 \mathrm{~h}$. After cooling down to room temperature PCN-160 crystals were harvested.

Synthesis of PCN-160(Zr)@Zn2(BDC)2(TED) and PCN-160(Zr)@PS. The hierarchical MOF-on-MOF composites were synthesized on the basis of previous reports with slight modifications. ${ }^{[5]}$ Activated PCN-160 (0.012 g) was immersed in a solution of BDC $(0.084 \mathrm{~g})$ in $3 \mathrm{~mL}$ DMF in a Pyrex vial. The mixture was heated in a $100{ }^{\circ} \mathrm{C}$ oven for $6 \mathrm{~h}$. A solution of TED $(0.028 \mathrm{~g})$ and $\mathrm{Zn}\left(\mathrm{NO}_{3}\right)_{2} \cdot 9 \mathrm{H}_{2} \mathrm{O}(0.150 \mathrm{~g})$ in 3 $\mathrm{mL} \mathrm{DMF}$ were further added into the vial. The mixture was heated in a $100{ }^{\circ} \mathrm{C}$ oven for 2 days to generate the crystals of PCN-160@ $\mathrm{Zn}_{2}(\mathrm{BDC})_{2}$ (TED). The solution was decanted, and the crystals were repeatedly washed with DMF. The two-step preparation procedures from PCN-160(Zr)@ $\mathrm{Zn}_{2}(\mathrm{BDC})_{2}$ (TED) and PCN-160(Zr)@PS were described above, including polymerization of styrene monomers in hierarchical MOFs and template removal by Na-EDTA solutions. 


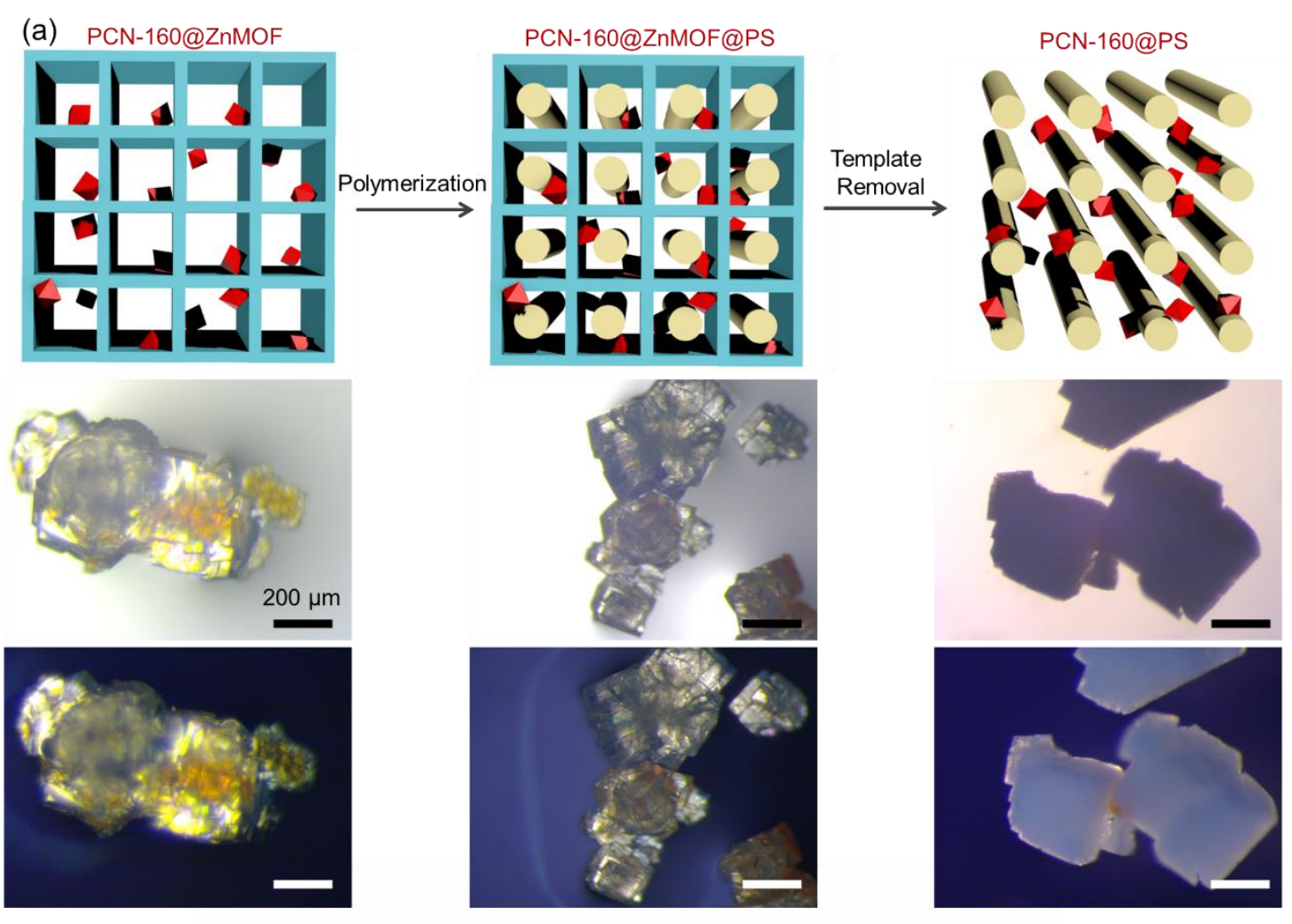

(b)

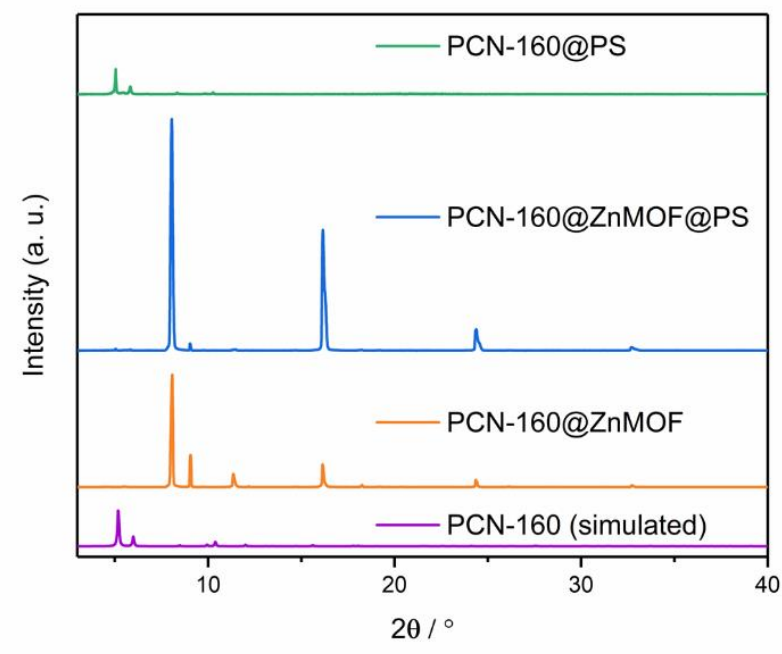

Figure S20. (a) Models and optical images showing the stepwise polymerization and template removal processes in hierarchical PCN-160@Z $\mathrm{Zn}_{2}(\mathrm{BDC})_{2}$ (TED). (b) PXRD

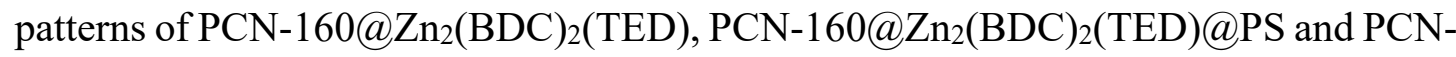
$160 @$ PS. (Cyan frameworks $=\mathrm{Zn}_{2}(\mathrm{BDC})_{2}(\mathrm{TED})$; yellow rods = PS; orange octahedra $=\mathrm{PCN}-160)$ 
Synthesis of PCN-224. PCN-224 was synthesized on the basis of previous reports with slight modifications. ${ }^{[7]} \mathrm{ZrOCl}_{2}(30 \mathrm{mg})$, TCPP $(10 \mathrm{mg})$, benzoic acid $(600 \mathrm{mg})$ and DMF $(3 \mathrm{~mL})$ were charged in a Pyrex vial. The mixture was heated in a $120{ }^{\circ} \mathrm{C}$ oven for $24 \mathrm{~h}$. After cooling down to room temperature PCN-224 were harvested.

Synthesis of PCN-224(Zr)@Zn2(BDC)2(TED) and PCN-224(Zr)@PS. The hierarchical MOF-on-MOF composites were synthesized on the basis of previous reports with slight modifications. ${ }^{[5]}$ Activated PCN-224 (0.012 g) was immersed in a solution of BDC $(0.084 \mathrm{~g})$ in $3 \mathrm{~mL}$ DMF in a Pyrex vial. The mixture was heated in a $100{ }^{\circ} \mathrm{C}$ oven for $6 \mathrm{~h}$. A solution of TED $(0.028 \mathrm{~g})$ and $\mathrm{Zn}\left(\mathrm{NO}_{3}\right)_{2} \cdot 9 \mathrm{H}_{2} \mathrm{O}(0.150 \mathrm{~g})$ in 3 $\mathrm{mL}$ DMF were further added into the vial. The mixture was heated in a $100^{\circ} \mathrm{C}$ oven for 2 days to generate the crystals of PCN-224@ $\mathrm{Zn}_{2}(\mathrm{BDC})_{2}$ (TED). The solution was decanted, and the crystals were repeatedly washed with DMF. The two-step preparation procedures from PCN-224(Zr)@Zn 2 (BDC) 2 (TED) and PCN-224(Zr)@PS were described above, including polymerization of styrene monomers in hierarchical MOFs and template removal by Na-EDTA solutions. 

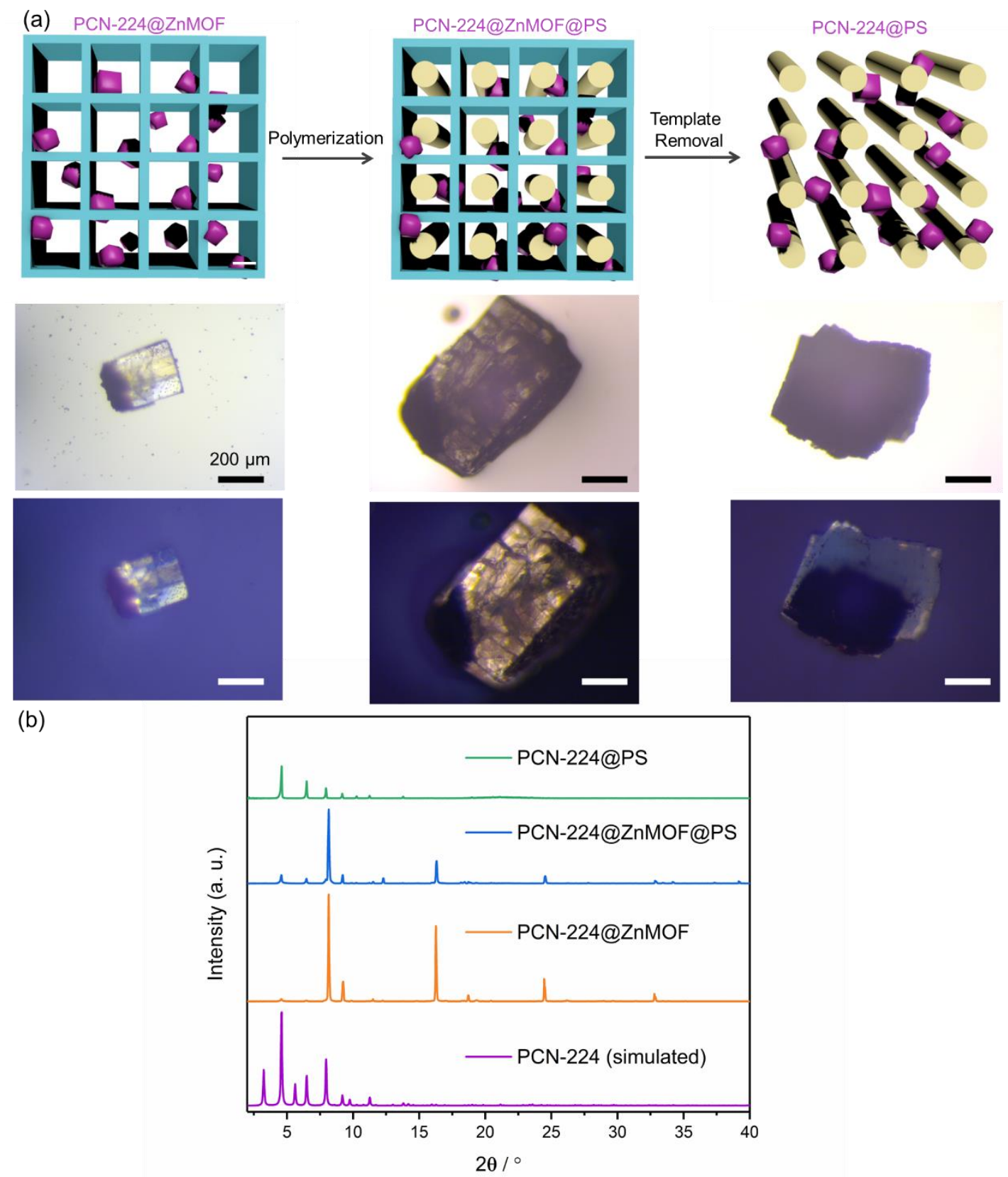

Figure S21. (a) Models and optical images showing the stepwise polymerization and template removal processes in hierarchical PCN-224@Zn $\mathrm{Zn}_{2}(\mathrm{BDC})_{2}$ (TED). (b) PXRD patterns of PCN-224@Zn 2 (BDC) 2 (TED), PCN-224@ $\mathrm{Zn}_{2}(\mathrm{BDC})_{2}(\mathrm{TED}) @ \mathrm{PS}$ and PCN$224 @$ PS. $\left(\right.$ Cyan frameworks $=\mathrm{Zn}_{2}(\mathrm{BDC})_{2}(\mathrm{TED})$; yellow rods = PS; purple cubes = $\mathrm{PCN}-224)$ 

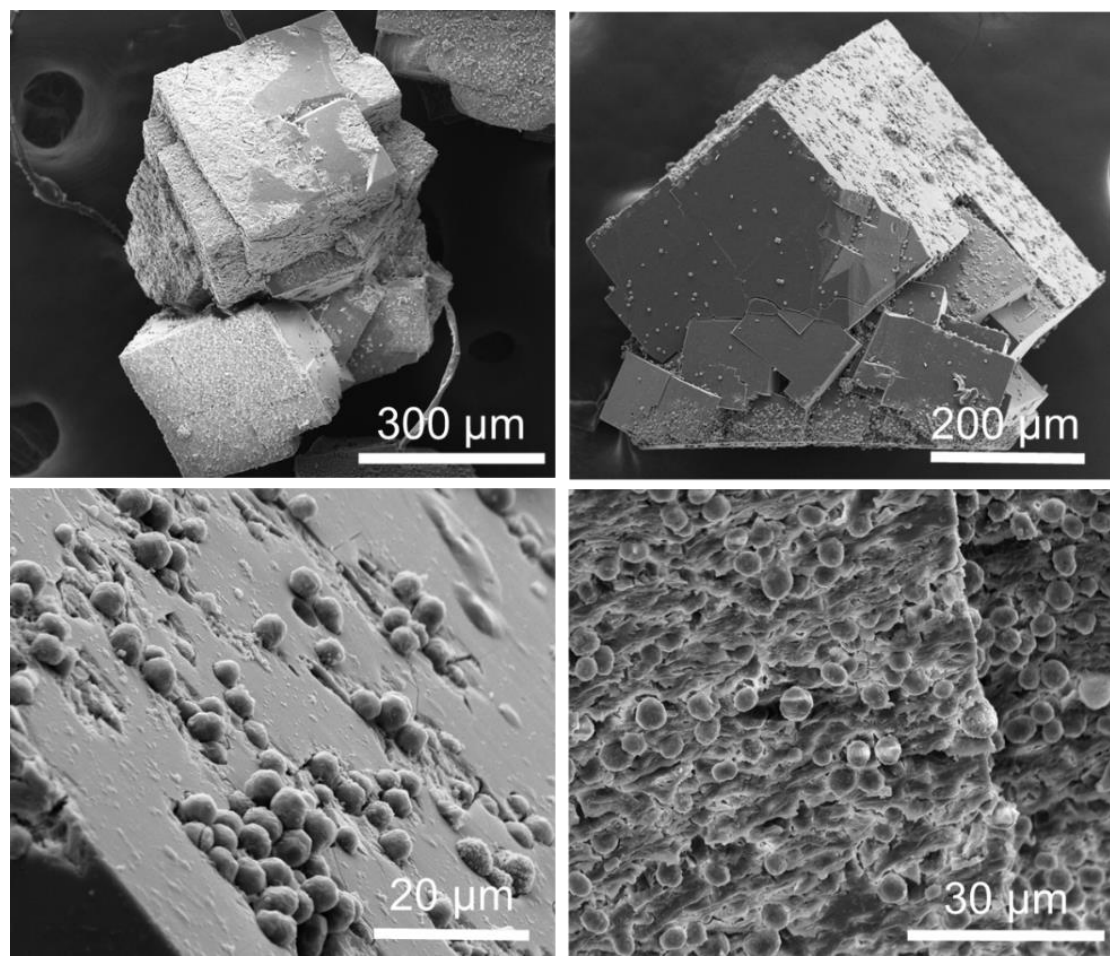

Figure S22. SEM images of PCN-224@Zn $2(\mathrm{BDC})_{2}$ (TED)@PS showing the dispersion of spherical PCN-224 in the matrix of $\mathrm{Zn}_{2}(\mathrm{BDC})_{2}$ (TED)@PS.
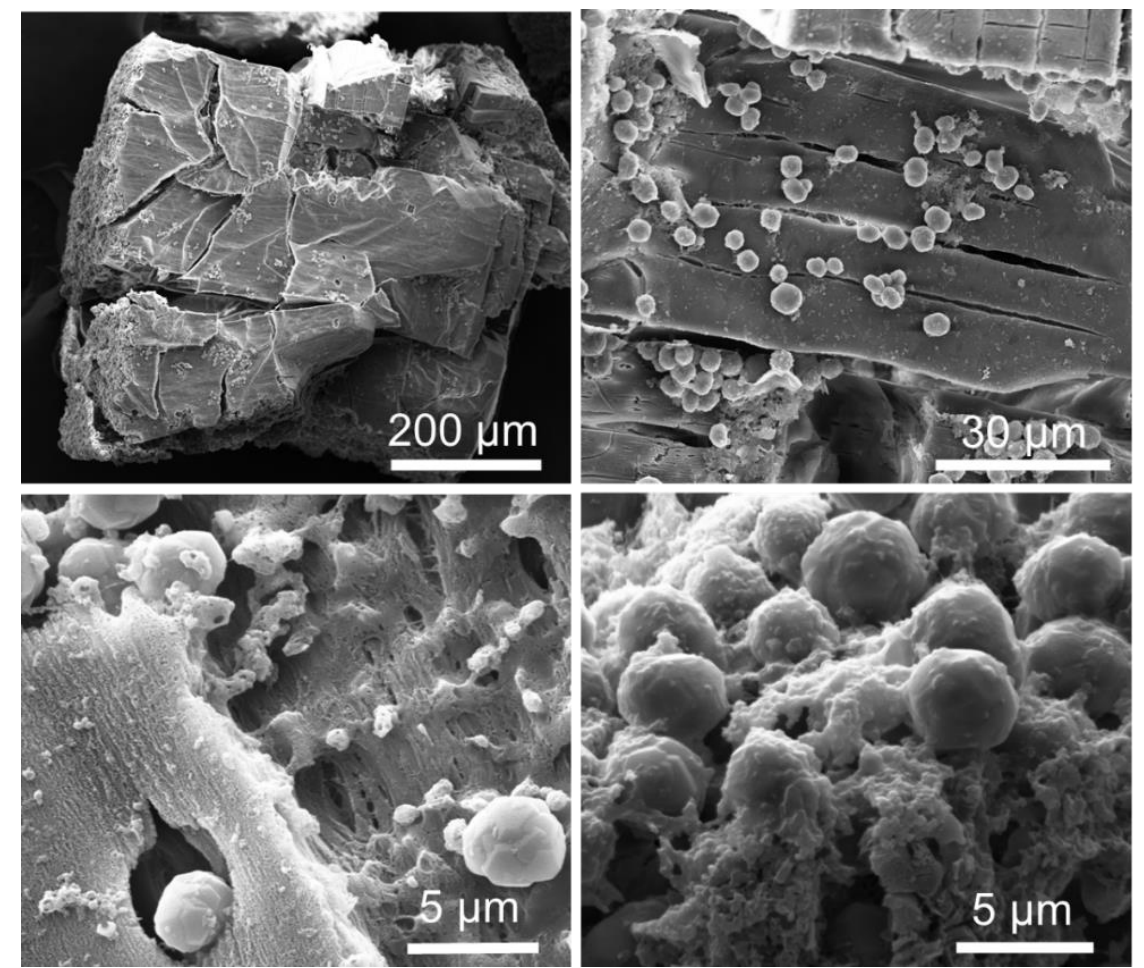

Figure S23. SEM images of PCN-224@PS after template removal showing the maintained moprhlogy of MOF-on-polymer architectures. The presence of cracks were caused by the mechanistic lability of polymer phases under vacuum in the SEM 
observation.

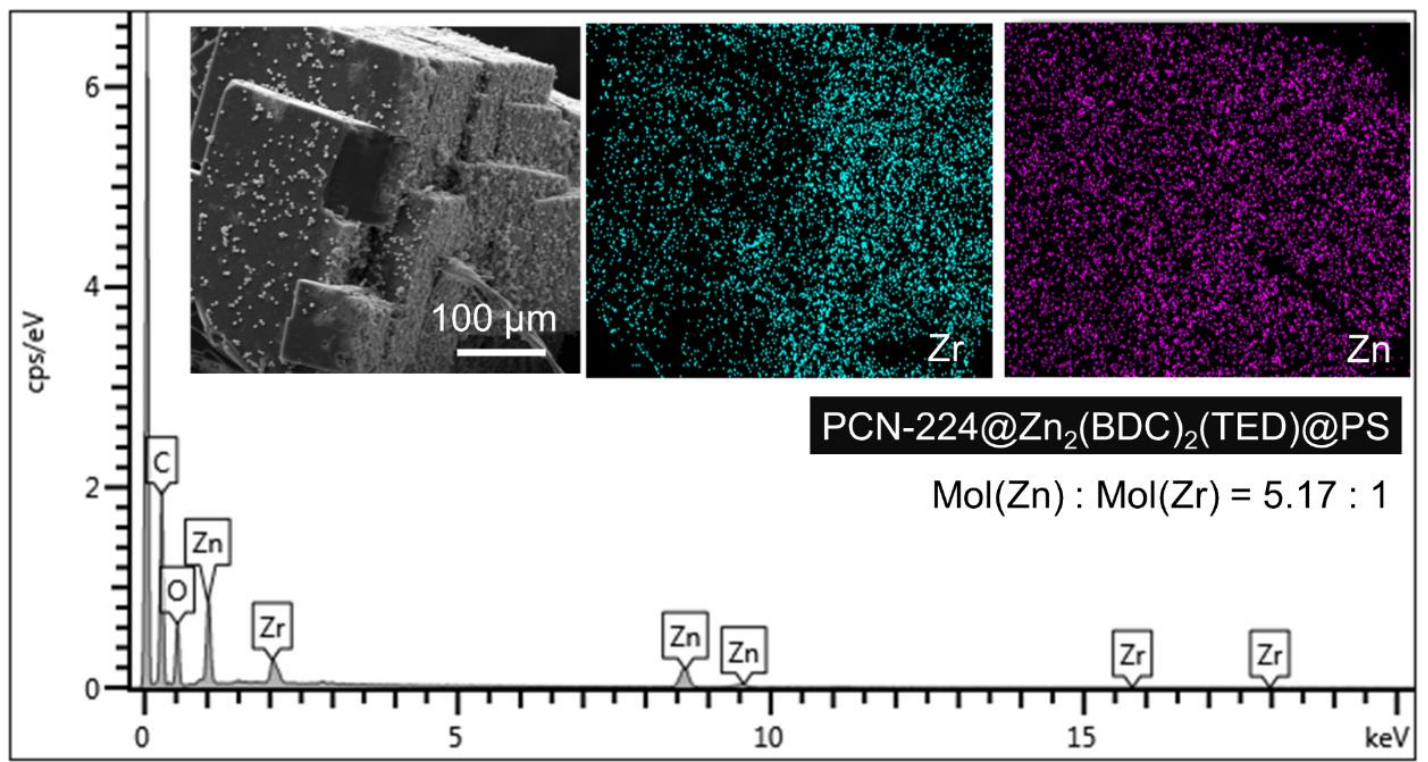

Figure S24. SEM images, the corresponding EDS mapping and EDS spectrum of PCN224@Zn2(BDC)2(TED)@PS.

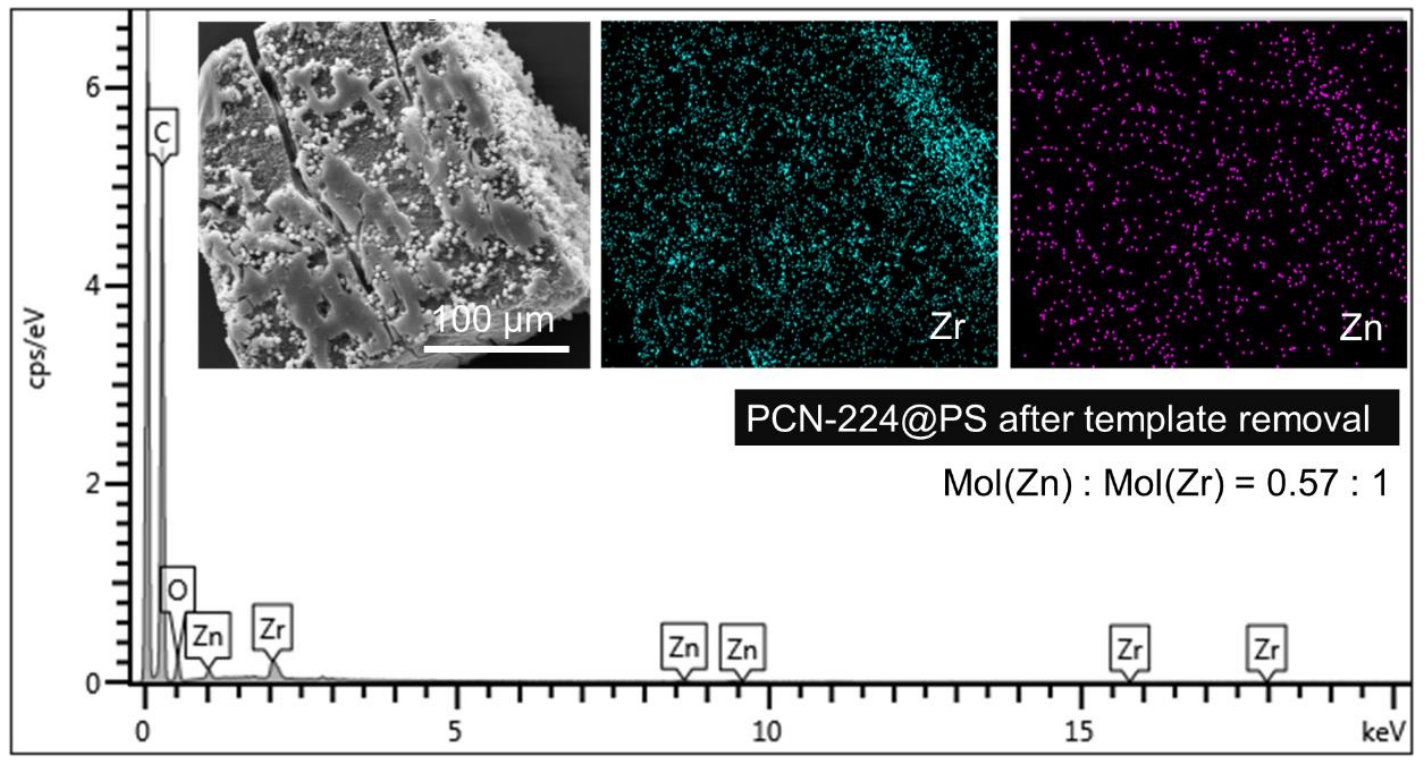

Figure S25. SEM images, the corresponding EDS mapping and EDS spectrum of PCN224@PS showing that most of the Zn ions are removed from the composites after NaEDTA treatment. 
Synthesis of MOF-801. MOF-801 was synthesized according to the literature with slight modification. ${ }^{[8]} \mathrm{ZrOCl}_{2} \cdot 8 \mathrm{H}_{2} \mathrm{O}(100 \mathrm{mg})$, fumaric acid $(75 \mathrm{mg}), \mathrm{HAc}(10 \mathrm{~mL})$ and DMF $(10 \mathrm{~mL})$ were charged in a Pyrex vial. The mixture was heated in $120^{\circ} \mathrm{C}$ oven for 2 days. After cooling down to room temperature, the resulted MOF-801 was separated by centrifugation and washed several times with DMF.

Synthesis of MOF-801(Zr)@Zn2(BDC)2(TED) and MOF-801(Zr)@PS. The hierarchical MOF-on-MOF composites were synthesized on the basis of previous reports with slight modifications. ${ }^{[5]}$ Activated MOF-801 (0.010 g) was immersed in a solution of BDC $(0.084 \mathrm{~g})$ in $3 \mathrm{~mL}$ DMF in a Pyrex vial. The mixture was heated in a $100{ }^{\circ} \mathrm{C}$ oven for $6 \mathrm{~h}$. A solution of TED $(0.028 \mathrm{~g})$ and $\mathrm{Zn}\left(\mathrm{NO}_{3}\right)_{2} \cdot 9 \mathrm{H}_{2} \mathrm{O}(0.150 \mathrm{~g})$ in 3 $\mathrm{mL} \mathrm{DMF}$ were further added into the vial. The mixture was heated in a $100{ }^{\circ} \mathrm{C}$ oven for 2 days to generate the crystals of MOF-801@ $\mathrm{Zn}_{2}(\mathrm{BDC})_{2}$ (TED). The solution was decanted, and the crystals were repeatedly washed with DMF. The two-step preparation procedures from MOF-801(Zr)@Zn 2 (BDC)2(TED) and MOF-801(Zr)@PS were described above, including polymerization of styrene monomers in hierarchical MOFs and template removal by Na-EDTA solutions. 


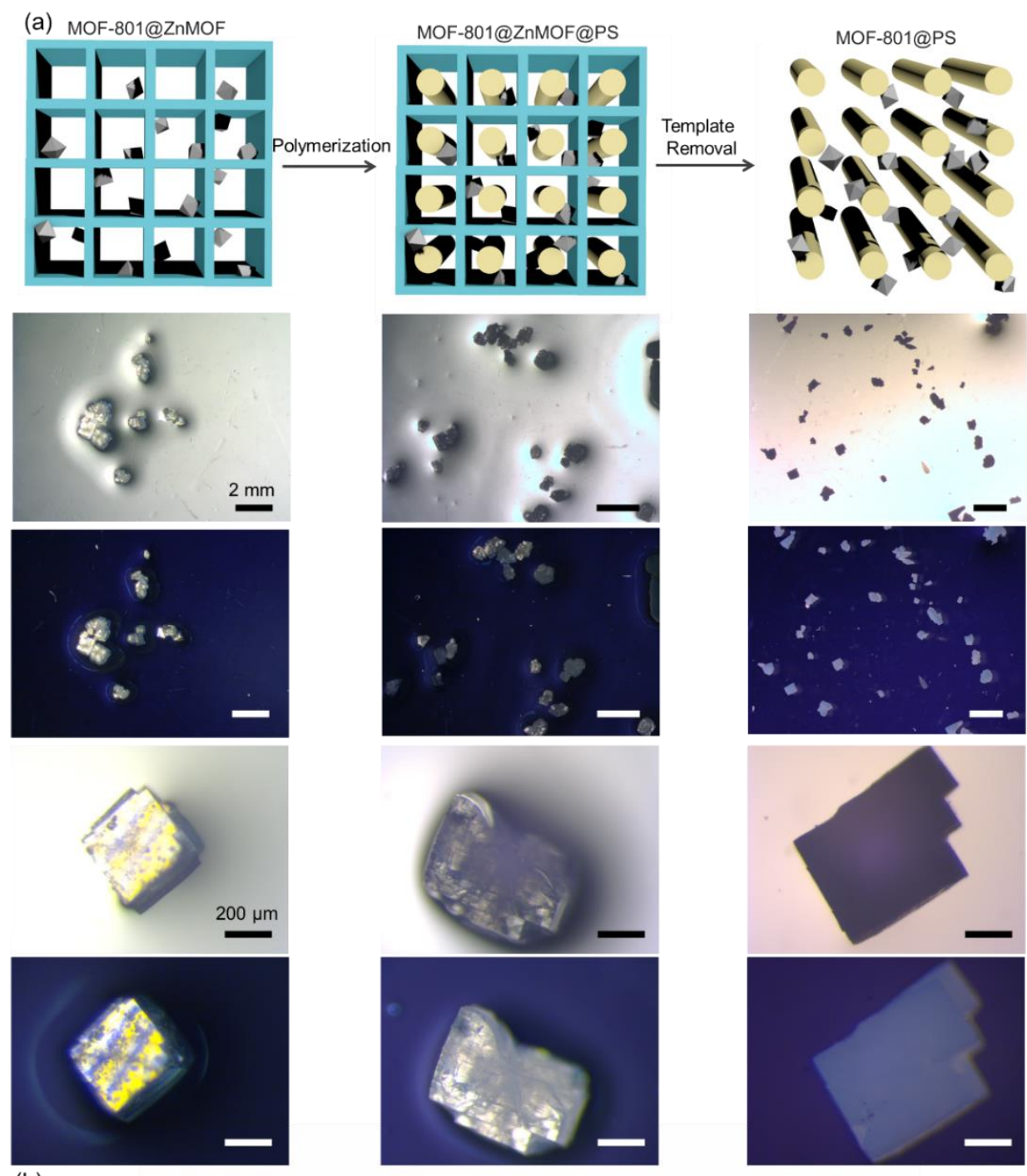

(b)

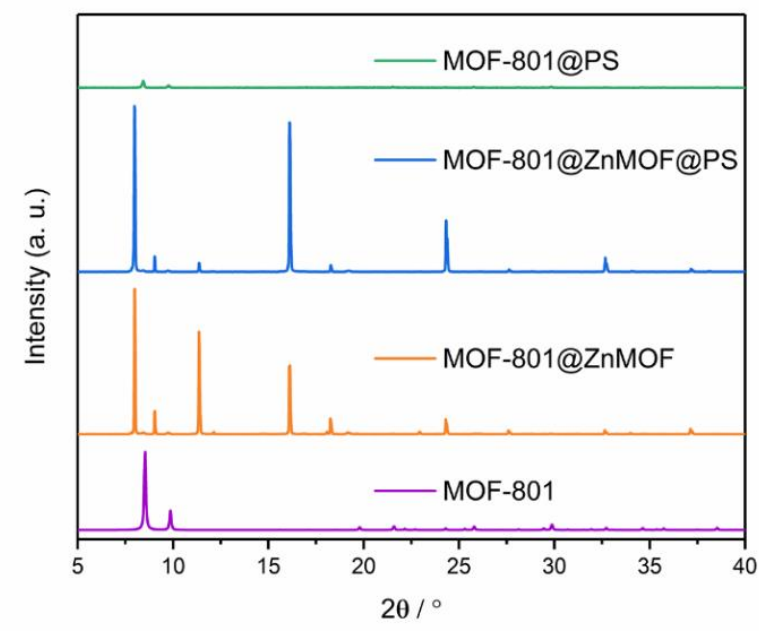

Figure S26. (a) Models and optical images showing the stepwise polymerization and template removal processes in hierarchical MOF-801@ $\mathrm{Zn}_{2}(\mathrm{BDC})_{2}$ (TED). (b) PXRD

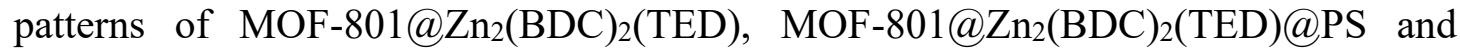
MOF-801@PS. (Cyan frameworks $=\mathrm{Zn}_{2}(\mathrm{BDC})_{2}(\mathrm{TED})$; yellow rods = PS; grey octahedra $=$ MOF-801) 
Synthesis of PCN-900(Eu). PCN-900 was synthesized according to the literature with slight modification. ${ }^{[9]}$ A mixture of TCPP (30 mg), Eu( $\left.\mathrm{NO}_{3}\right)_{3} \cdot 6 \mathrm{H}_{2} \mathrm{O}$ (100 mg), DCDPS $(500 \mathrm{mg})$ and 2-fluorobenzoic acid $(8 \mathrm{~g})$ was added to a $40 \mathrm{~mL}$ glass scintillation vial containing $30 \mathrm{~mL}$ DMF. After 10 mins of sonication, the vial was sealed and then placed into a preheated oven at $120^{\circ} \mathrm{C}$ for 3 days. The dark-red single crystals of PCN-900(Eu) were filtered and obtained.

Synthesis of PCN-900(Eu)@Zn 2 (BDC)2(TED) and PCN-900(Eu)@PS. The hierarchical MOF-on-MOF composites were synthesized on the basis of previous reports with slight modifications. ${ }^{[5]}$ Activated PCN-900 (0.010 g) was immersed in a solution of BDC $(0.084 \mathrm{~g})$ in $3 \mathrm{~mL}$ DMF in a Pyrex vial. The mixture was heated in a $100{ }^{\circ} \mathrm{C}$ oven for $6 \mathrm{~h}$. A solution of TED $(0.028 \mathrm{~g})$ and $\mathrm{Zn}\left(\mathrm{NO}_{3}\right)_{2} \cdot 9 \mathrm{H}_{2} \mathrm{O}(0.150 \mathrm{~g})$ in 3 $\mathrm{mL}$ DMF were further added into the vial. The mixture was heated in a $100^{\circ} \mathrm{C}$ oven for 2 days to generate the crystals of PCN-900@ $\mathrm{Zn}_{2}(\mathrm{BDC})_{2}$ (TED). The solution was decanted, and the crystals were repeatedly washed with DMF. The two-step preparation procedures from PCN-900@Z $\mathrm{Zn}_{2}(\mathrm{BDC})_{2}$ (TED) and PCN-900@PS were described above, including polymerization of styrene monomers in hierarchical MOFs and template removal by Na-EDTA solutions. 

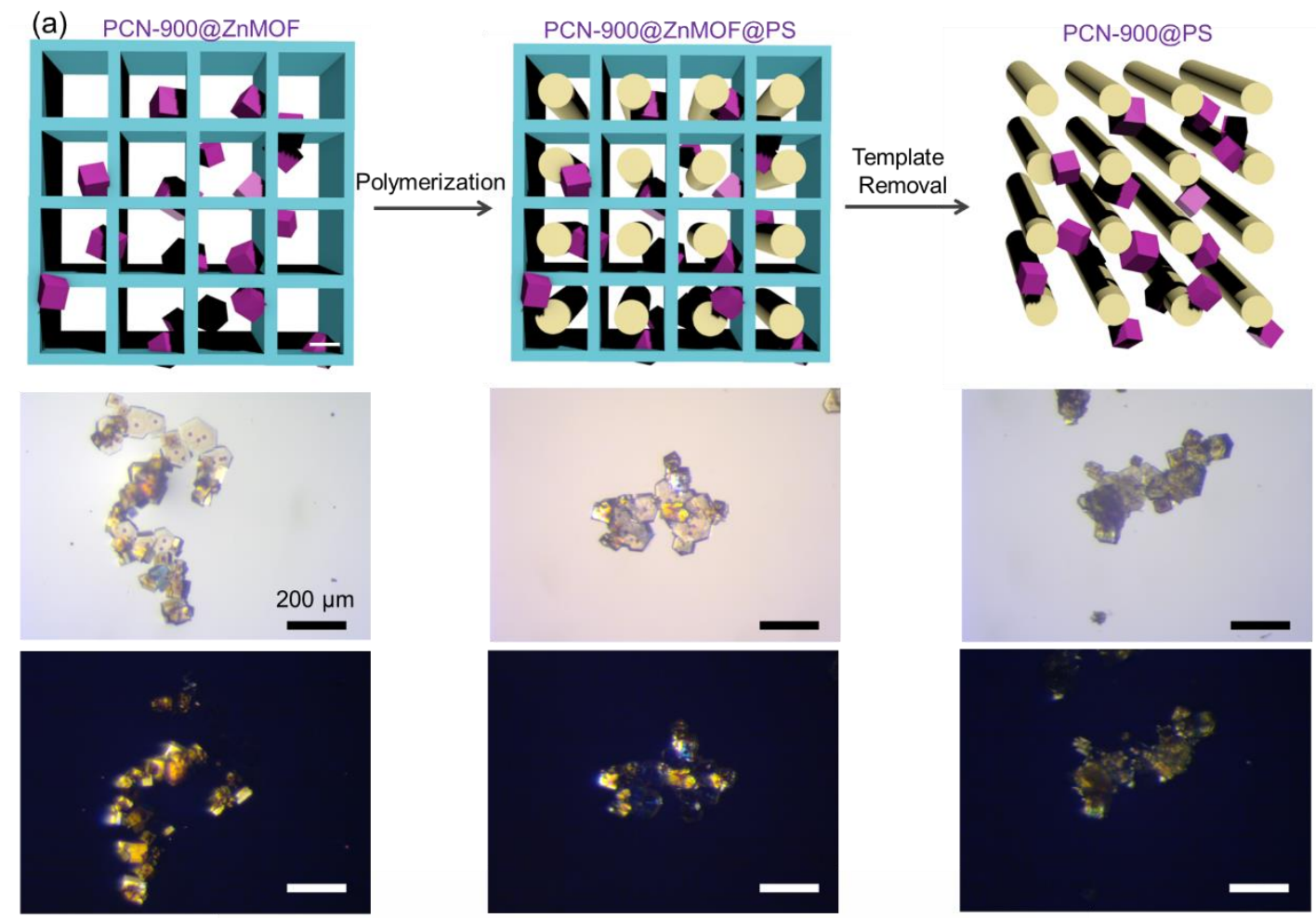

(b)

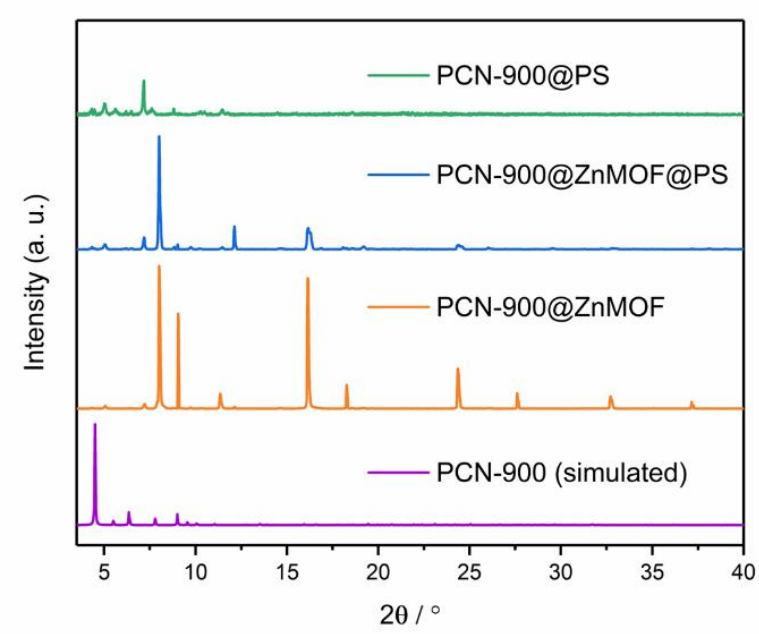

Figure S27. (a) Models and optical images showing the stepwise polymerization and template removal processes in hierarchical PCN-900@Z $\mathrm{Zn}_{2}(\mathrm{BDC})_{2}$ (TED). (b) PXRD

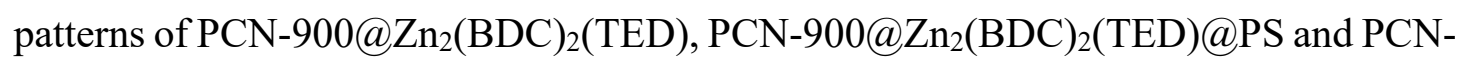
900@PS. (Cyan frameworks $=\mathrm{Zn}_{2}(\mathrm{BDC})_{2}(\mathrm{TED})$; yellow rods $=\mathrm{PS}$; prune cubes $=$ PCN-900) 
Synthesis of COF-303. COF-303 was synthesized on the basis of a previous report. ${ }^{[10]}$ A vial was charged with tetrakis(4-formylphenyl)methane (TFM, $10.8 \mathrm{mg}, 0.025$ mmol), aniline ( $0.12 \mathrm{~mL}, 52$ equiv.), and $0.25 \mathrm{~mL}$ of 1,4-dioxane, then $0.1 \mathrm{~mL}$ of aqueous acetic acid (6 M) was added to the solution. Phenylenediamine (PDA, $5.4 \mathrm{mg}$, $0.05 \mathrm{mmol})$ dissolved in 1,4-dioxane $(0.25 \mathrm{~mL})$ was then added. Then the mixture was allowed to further stand at ambient temperature. The single crystals of COF-303 then slowly crystallized out at ambient temperature in 15 days.

Synthesis of COF-303@Zn2(BDC)2(TED) and COF-303@PS. The hierarchical COF-on-MOF composites were synthesized on the basis of previous reports with slight modifications. ${ }^{[11]}$ COF-303 (0.005 g) was immersed in a solution of BDC (0.084 g) in $3 \mathrm{~mL}$ DMF in a Pyrex vial. The mixture was heated in a $100^{\circ} \mathrm{C}$ oven for $2 \mathrm{~h}$. A solution of TED $(0.028 \mathrm{~g})$ and $\mathrm{Zn}\left(\mathrm{NO}_{3}\right)_{2} \cdot 9 \mathrm{H}_{2} \mathrm{O}(0.150 \mathrm{~g})$ in $3 \mathrm{~mL}$ DMF were further added into the vial. The mixture was heated in a $100{ }^{\circ} \mathrm{C}$ oven for 2 days to generate the crystals of COF-303@ $\mathrm{Zn}_{2}(\mathrm{BDC})_{2}$ (TED). The solution was decanted, and the crystals were repeatedly washed with DMF. The two-step preparation procedures from COF303@ $\mathrm{Zn}_{2}(\mathrm{BDC})_{2}(\mathrm{TED})$ and COF-303@PS were described above, including polymerization of styrene monomers in hierarchical MOFs and template removal by Na-EDTA solutions.

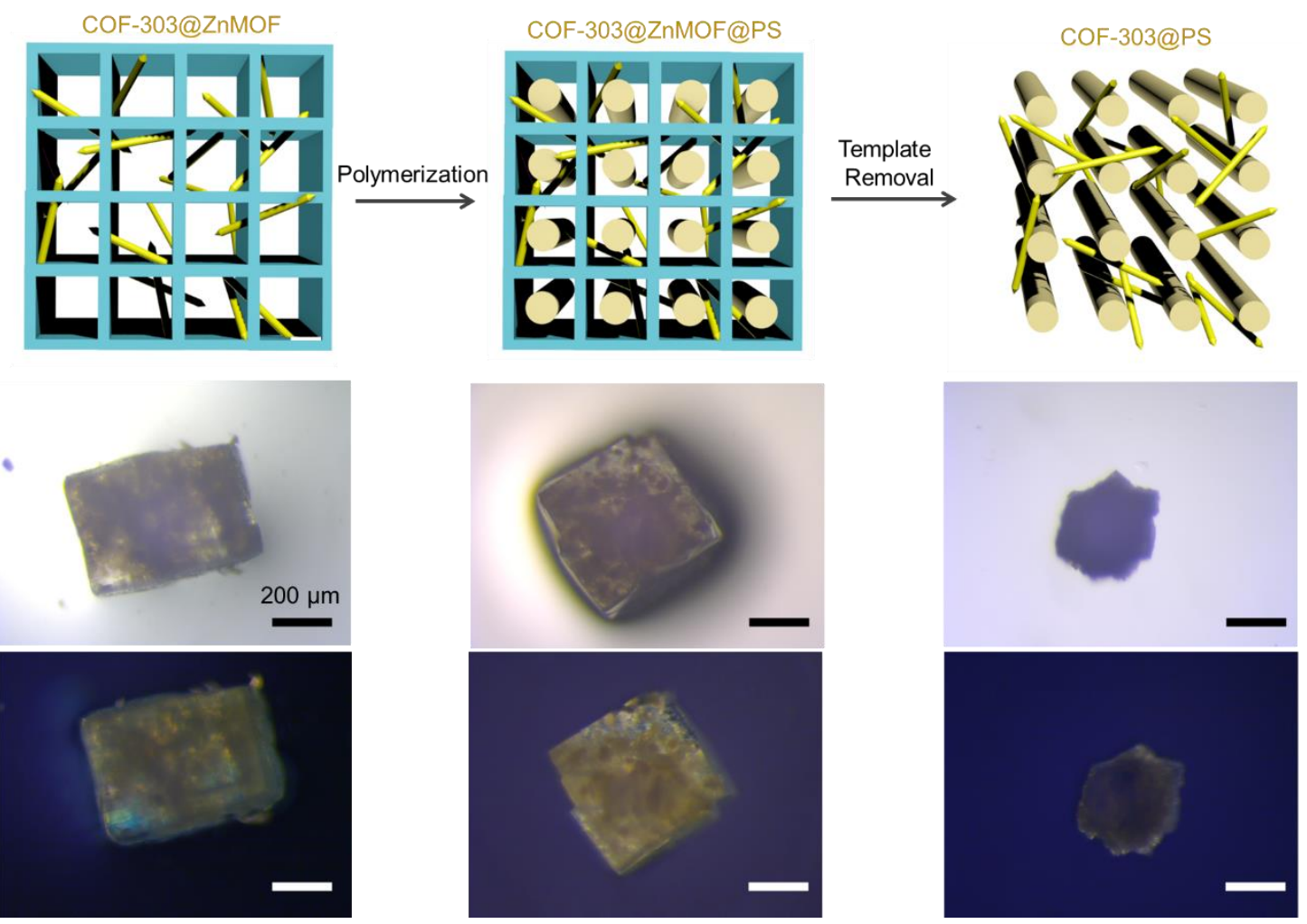

Figure S28. Models and optical images showing the stepwise polymerization and template removal processes in hierarchical COF-303@ $\mathrm{Zn}_{2}(\mathrm{BDC})_{2}(\mathrm{TED})$. (Cyan frameworks $=\mathrm{Zn}_{2}(\mathrm{BDC})_{2}(\mathrm{TED}) ;$ yellow rods $=\mathrm{PS} ;$ yellow needles $\left.=\mathrm{COF}-303\right)$ 
Preparation of samples for gel permeation chromatography (GPC). Around $5 \mathrm{mg}$ of samples were immersed in $2 \mathrm{~mL} 40 \% \mathrm{HF}$ for $3 \mathrm{~h}$ to fully digest the MOF portions. The excess acid and water were removed under vacuum. Then $2 \mathrm{~mL}$ THF was added to dissolve the polymer for $1 \mathrm{~h}$. The solution was filtered through $1 \mu \mathrm{m}$ membrane before being injected into the GPC instrument.

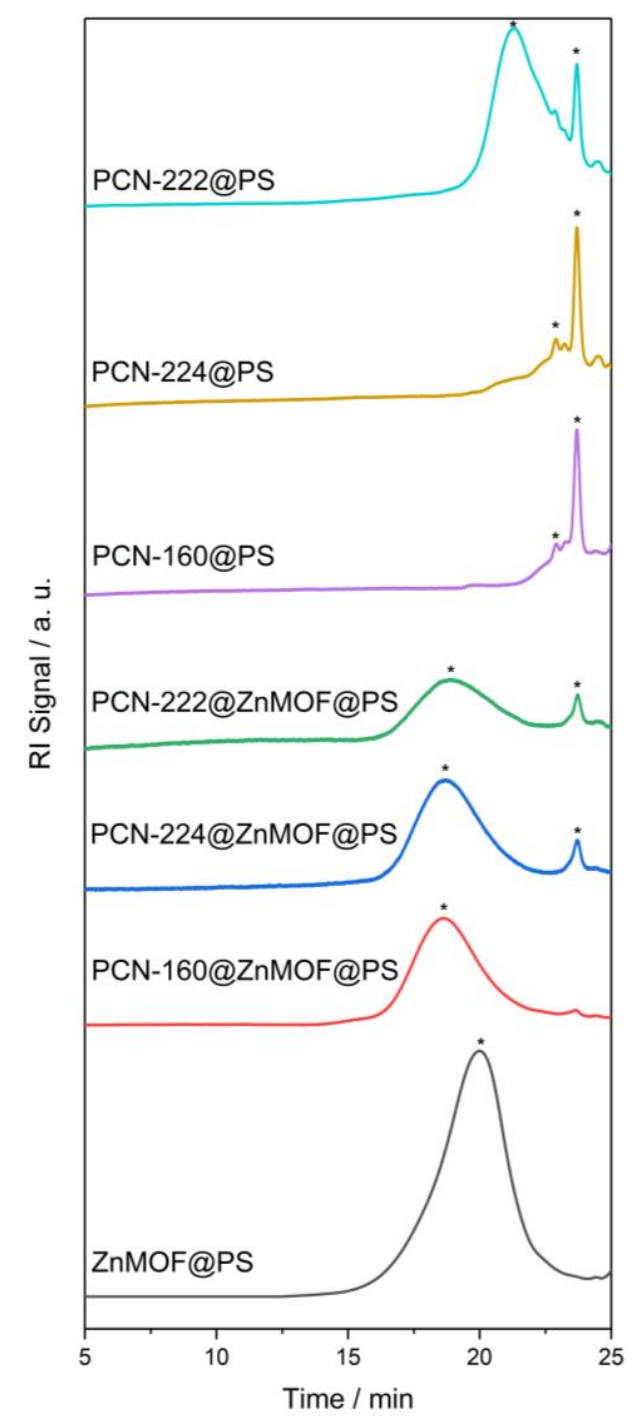

Figure S29. Gel permeation chromatogram of the synthesized PS, which was produced after framework removal of templating MOFs. The calculated number average molar mass $\mathrm{M}_{\mathrm{n}}$ and dispersity (Đ) values are listed in Table $\mathrm{S} 1$. 
Table S1. The calculated number average molar mass $M_{n}$ and dispersity (Đ) values of PS produced from different templating MOFs.

\begin{tabular}{ccccc}
\hline Template & \multicolumn{2}{c}{ Peak 1 } & \multicolumn{2}{c}{ Peak 2 } \\
& $\mathrm{M}_{\mathrm{n}}$ & $\mathrm{D}$ & $\mathrm{M}_{\mathrm{n}}$ & $\mathrm{D}$ \\
\hline ZnMOF & 11,453 & 3.869 & - & - \\
PCN-160@ZnMOF & 25,712 & 1.889 & - & - \\
PCN-224@ZnMOF & 23,921 & 2.019 & 905 & 1.006 \\
PCN-222@ZnMOF & 21,001 & 2.122 & 882 & 1.010 \\
PCN-160 & 1,641 & 1.009 & 902 & 1.004 \\
PCN-224 & 1,939 & 1.122 & 898 & 1.005 \\
PCN-222 & 4,320 & 1.374 & 897 & 1.005
\end{tabular}

Polymerization of vinyl monomers in PCN-222 and PCN-224. To further study the porosity of the MOF@polymer composites, polymerization was performed on the PCN-222 and PCN-224. The activated PCN-222 or PCN-224 (100 mg) was prepared by evacuation $(<0.1 \mathrm{kPa})$ at $130{ }^{\circ} \mathrm{C}$ for 1 day in a Pyrex reaction tube. Subsequently, the MOF was immersed in a purified monomer $(0.5 \mathrm{~mL})$ with AIBN $(3 \mathrm{mg})$ at room temperature for $0.5 \mathrm{~h}$ to incorporate the monomer and the radical initiator into the pores. After excess monomer external to the host crystals was removed completely by evacuation (St: $0.2 \mathrm{kPa}, \mathrm{MMA}: 2.0 \mathrm{kPa}$ ) at room temperature for $0.5 \mathrm{~h}$, the reaction tube was filled with nitrogen, and heated to $70{ }^{\circ} \mathrm{C}$ to perform the polymerization of $\mathrm{St}$ for $48 \mathrm{~h}$. The collected hierarchical MOF-on-polymer composites were collected and stored under a reduced pressure at room temperature. 

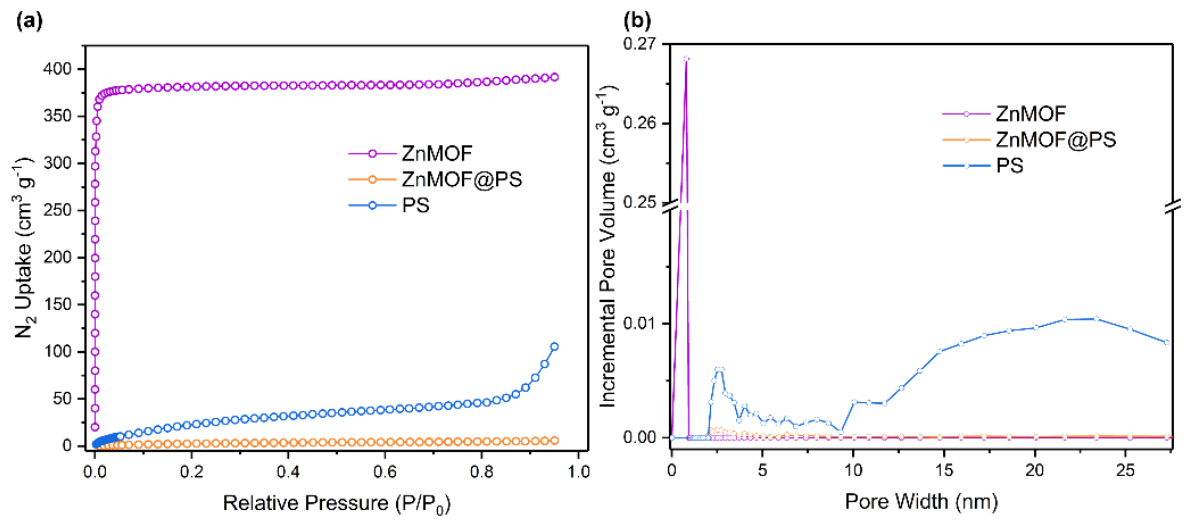

(c)

(d)
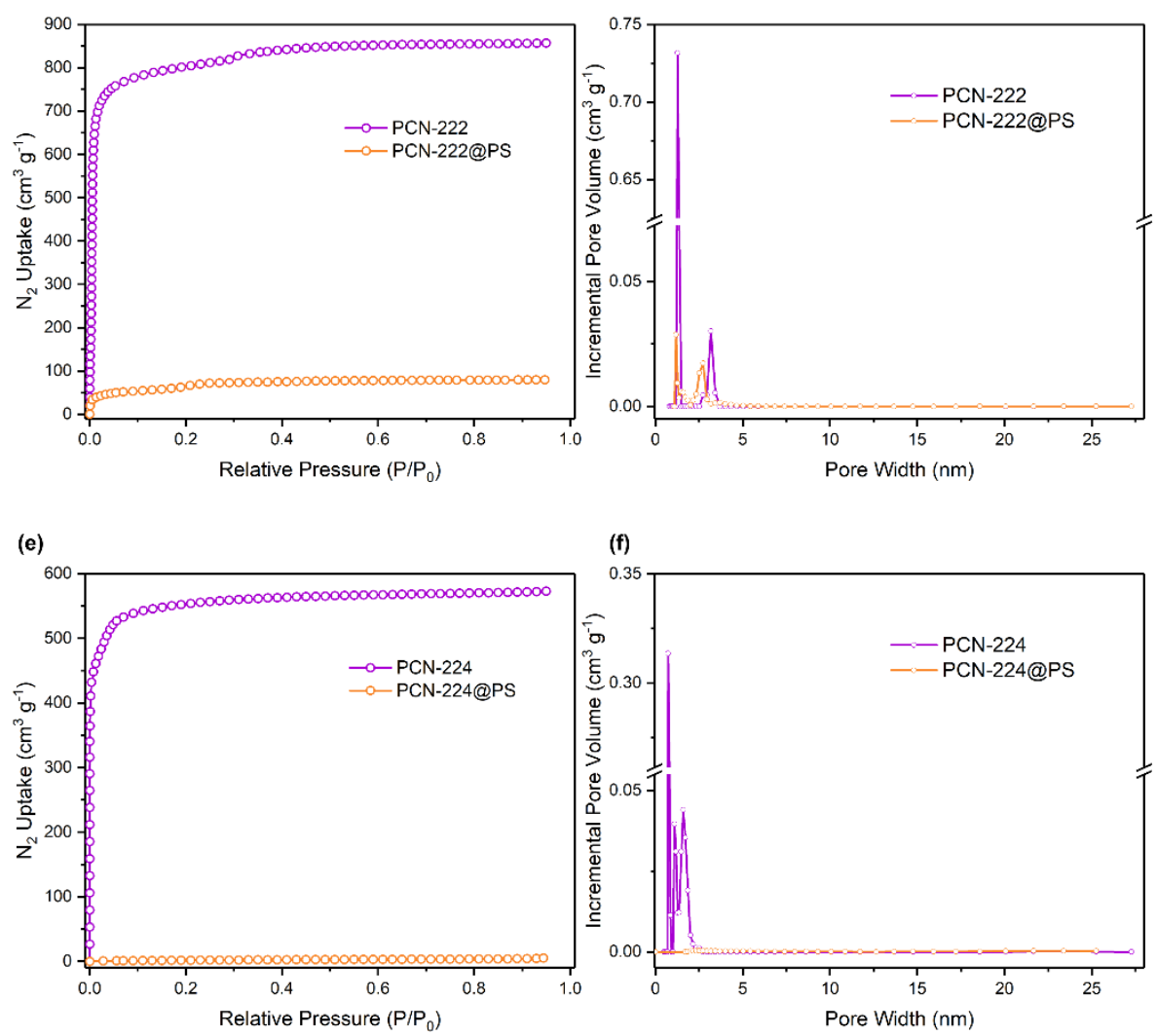

Figure S30. Nitrogen sorption tests and pore size distribution of MOFs, MOF@polymer composites, and polymers. The $\mathrm{Zn}_{2}(\mathrm{BDC})_{2}$ (TED) templated PS features mesopores. The surface areas of PCN-222 and PCN-224 will dramatically drop after polymerization, indicating the appearance of polymer chains within the pores. 
Table S2. Elemental contents in the MOF@polymer composite materials determined by ICP-MS.

\begin{tabular}{|c|c|c|}
\hline Sample & $\begin{array}{c}\mathrm{Zn}^{66}: \mathrm{Zr}^{90} \text { before } \\
\text { template removal }\end{array}$ & $\begin{array}{c}\mathrm{Zn}^{66}: \mathrm{Zr}^{90} \text { after } \\
\text { template removal }\end{array}$ \\
\hline PCN-2221low @ Zn 2 (BDC)2(TED)@PS & 39.7 & 0.200 \\
\hline PCN-222 med $_{2} \mathrm{Zn}_{2}(\mathrm{BDC})_{2}(\mathrm{TED}) @ \mathrm{PS}$ & 16.9 & 0.099 \\
\hline PCN-222high @ Zn 2 (BDC)2 2 (TED)@PS & 7.8 & 0.050 \\
\hline PCN-224@ Zn 2 (BDC) $)_{2}$ (TED)@PS & 12.3 & 0.103 \\
\hline PCN-160@ Zn $2(B D C)_{2}(T E D) @ P S$ & 20.1 & 0.073 \\
\hline PCN-801@ Zn 2 (BDC) $)_{2}(\mathrm{TED}) @ \mathrm{PS}$ & 33.2 & 0.130 \\
\hline
\end{tabular}




\section{References}

1. T. Uemura, T. Kaseda, S. Kitagawa, Chem. Mater. 2013, 25, 3772-3776.

2. a) M. L. Kelty, W. Morris, A. T. Gallagher, J. S. Anderson, K. A. Brown, C. A. Mirkin, T. D. Harris, Chem. Comm. 2016, 52, 7854-7857; b) S. Wang, Y. Chen, S. Wang, P. Li, C. A. Mirkin, O. K. Farha, J. Am. Chem. Soc. 2019, 141, 2215-2219.

3. D. Feng, Z. Y. Gu, J. R. Li, H. L. Jiang, Z. Wei, H.-C. Zhou, Angew. Chem. Int. Ed. 2012, 51, 10307-10310.

4. X. Gong, Y. Shu, Z. Jiang, L. Lu, X. Xu, C. Wang, H. Deng, Angew. Chem. Int. Ed. 2020, 59, 5326-5331.

5. L. Feng, S. Yuan, J. L. Li, K. Y. Wang, G. S. Day, P. Zhang, Y. Wang, H.-C. Zhou, ACS Cent. Sci. 2018, 4, 1719-1726.

6. S. A. Yuan, L. F. Zou, J. S. Qin, J. L. Li, L. Huang, L. A. Feng, X. A. Wang, M. Bosch, A. Alsalme, T. Cagin, H.-C. Zhou, Nat. Commun. 2017, 8, 15356.

7. D. W. Feng, W. C. Chung, Z. W. Wei, Z. Y. Gu, H. L. Jiang, Y. P. Chen, D. J. Darensbourg, H.C. Zhou, J. Am. Chem. Soc. 2013, 135, 17105-17110.

8. L. Feng, S. Yuan, L. L. Zhang, K. Tan, J. L. Li, A. Kirchon, L. M. Liu, P. Zhang, Y. Han, Y. J. Chabal, H.-C. Zhou, J. Am. Chem. Soc. 2018, 140, 2363-2372.

9. L. Zhang, S. Yuan, L. Feng, B. Guo, J. S. Qin, B. Xu, C. Lollar, D. Sun, H.-C. Zhou, Angew. Chem. Int. Ed. 2018, 57, 5095-5099.

10. T. Q. Ma, E. A. Kapustin, S. X. Yin, L. Liang, Z. Y. Zhou, J. Niu, L. H. Li, Y. Y. Wang, J. Su, J. Li, X. G. Wang, W. D. Wang, W. Wang, J. L. Sun, O. M. Yaghi, Science 2018, 361, 48-52.

11. L. Feng, K.-Y. Wang, X.-L. Lv, T.-H. Yan, J.-R. Li, H.-C. Zhou, J. Am. Chem. Soc. 2020, 142, 3069-3076. 